\title{
R\&D Cooperatives and Market Collusion: A Global Dynamic Approach
}

\author{
Jeroen Hinloopen' \\ Grega Smrkolj2 \\ Florian Wagener ${ }^{3}$
}

1 Utrecht University, The Netherlands;

2 Newcastle University, United Kingdom;

3 Faculty of Economics and Business, University of Amsterdam, and Tinbergen Institute, The Netherlands. 
Tinbergen Institute is the graduate school and research institute in economics of Erasmus University Rotterdam, the University of Amsterdam and VU University Amsterdam.

More TI discussion papers can be downloaded at http://www.tinbergen.nl

Tinbergen Institute has two locations:

Tinbergen Institute Amsterdam

Gustav Mahlerplein 117

1082 MS Amsterdam

The Netherlands

Tel.: +31(0)20525 1600

Tinbergen Institute Rotterdam

Burg. Oudlaan 50

3062 PA Rotterdam

The Netherlands

Tel.: +31(0)10 4088900

Fax: +31(0)10 4089031 


\title{
R\&D Cooperatives and Market Collusion: A Global Dynamic Approach*
}

\author{
Jeroen Hinloopen $^{\dagger} \quad$ Grega Smrkolj ${ }^{\ddagger} \quad$ Florian Wagener $^{\S}$
}

June, 2016

\begin{abstract}
We present a continuous-time generalization of the seminal R\&D model of d'Aspremont and Jacquemin (American Economic Review, 1988) to examine the trade-off between the benefits of allowing firms to cooperate in R\&D and the corresponding increased potential for product market collusion. We consider all trajectories that are candidates for an optimal solution as well as initial marginal cost levels that exceed the choke price. Firms that collude develop further a wider range of initial technologies, pursue innovations more quickly, and are less likely to abandon a technology. Product market collusion could thus yield higher total surplus.
\end{abstract}

Keywords: Antitrust policy, Bifurcations, Collusion, R\&D cooperatives, Spillovers

JEL: D43, D92, L13, L41, O31, O38

\section{Introduction}

An important reason for allowing firms to set up $R \& D$ cooperatives is that these "organizations, jointly controlled by at least two participating entities, whose primary purpose is to engage in cooperative R\&D" (Caloghirou et al., 2003) internalize technological spillovers - the free flow of knowledge from the knowledge creator to its competitors. ${ }^{1}$ The exemption for R\&D cooperatives in anti-cartel legislation is thus perceived to diminish the failure of the market

\footnotetext{
${ }^{*}$ Thanks are due to Bernd Ebersberger, Morten Hviid, Maurizio Iacopetta, Corinne Langinier, Bruce Lyons, Stephen Martin, Jo Seldeslachts, Nan Yang, and to seminar participants at the Tinbergen Institute (Amsterdam, June 2011), at EARIE 2011 (Stockholm, September 2011), at CeNDEF, University of Amsterdam (Amsterdam, April 2012), at the Centre for Competition Policy at the University of East Anglia (Norwich, April 2012), at the 12th Viennese Workshop on Optimal Control, Dynamic Games and Nonlinear Dynamics (Vienna, June 2012), at the 14th International Schumpeter Society Conference (Brisbane, July 2012), at the Netherlands Economists Day (Amsterdam, October 2012), at OFCE, Skema Business School (Sophia Antipolis, May 2013), at IIOC 2013 (Boston, May 2013), and at CEA 2016 (Ottawa, June 2016) for constructive comments.

${ }^{\dagger}$ Utrecht University School of Economics; J.Hinloopen@uu.nl.

${ }^{\ddagger}$ Newcastle University Business School. Corresponding author. Address: Newcastle University Business School, 5 Barrack Road, Newcastle upon Tyne, NE1 4SE, United Kingdom; E-mail address: Grega.Smrkolj@newcastle.ac.uk.

${ }^{\S}$ University of Amsterdam and Tinbergen Institute; F.O.O.Wagener@uva.nl.

${ }^{1}$ Bloom et al. (2013) estimate that a $10 \%$ increase in a competitor's R\&D is associated with up to a $3.8 \%$ increase in a firm's own market value. Internalizing technological spillovers is one of the prime reasons for firms to join an R\&D cooperative (Hernan et al., 2003; see also Röller et al., 2007).
} 
for R\&D. ${ }^{2}$ However, as Scherer (1980) observes: "the most egregious price fixing schemes in American history were brought about by R\&D cooperatives", an observation that constitutes the classic counterargument to a permissive antitrust treatment of R\&D markets (Pfeffer and Nowak, 1976; Grossman and Shapiro, 1986; Brodley, 1990). ${ }^{3}$ At the same time, it is quite well established that the prospect of future market power enhances a firm's incentives to invest in R\&D. ${ }^{4}$ As Alan Greenspan (1962) puts it:

No one will ever know what new products, processes, machines, and cost-saving mergers failed to come into existence, killed by the Sherman Act before they were born. No one can ever compute the price that all of us have paid for that Act which, by inducing less effective use of capital, has kept our standard of living lower than would otherwise have been possible.

In this paper we develop a dynamic model of $R \& D$ that considers explicitly the cost of "new ... processes" that "failed to come into existence ... before they were born" because of the ban on price-fixing agreements.

The channels through which cooperation in $R \& D$ facilitates product market collusion have been examined in a number of theoretical studies (Martin, 1995; Greenlee and Cassiman, 1999; Cabral, 2000; Lambertini et al., 2002; Miyagiwa, 2009). According to Fisher (1990, p. 194):

... [firms] cooperating in R\&D will tend to talk about other forms of cooperation. Furthermore, in learning how other firms react and adjust in living with each other, each cooperating firm will get better at coordination. Hence, competition in the product market is likely to be harmed.

In the short run, the reduced intensity of product market competition is likely to hurt consumers. At the same time, it could enhance the functioning of an R\&D cooperative. For instance, Geroski (1992) argues that it is the feedback from product markets that directs research towards profitable tracks and that, therefore, for an innovation to be commercially successful, there must be strong ties between marketing and development of new products. And Jacquemin (1988) puts forward that R\&D cooperatives are fragile and unstable. He reasons that when there is no cooperation in the product market, there exists a continuous fear that one partner in the R\&D

\footnotetext{
${ }^{2}$ See Martin (1997) for an overview of the policy treatment of R\&D cooperatives in the E.U., the U.S., and Japan.

${ }^{3}$ Goeree and Helland (2008) find that in the U.S. the probability that firms join an R\&D cooperative has gone down due to a revision of antitrust leniency policy in 1993. This revision is perceived as making collusion less attractive. Goeree and Helland (2008) conclude that "Our results are consistent with RJVs [research joint ventures] serving, at least in part, a collusive function." Related evidence is reported by Duso et al. (2014). They find that the combined market share declines if partners in an RJV compete on the same product market ("horizontal RJVs"), while it increases if members of the RJV are not direct rivals ("vertical RJVs"). The laboratory experiments of Suetens (2008) show that members of an RJV are more likely to collude on price.

${ }^{4}$ The original observation is due to Schumpeter (1934, p. 82): "As soon as we go into the details and inquire into the individual items in which progress was most conspicuous, the trail leads not to the doors of those firms that work under conditions of comparatively free competition but precisely to the doors of the large concerns ... and a shocking suspicion dawns upon us that big business may have had more to do with creating that standard of living than with keeping it down." A formal treatment can be found in Tirole (1988).
} 
cooperative may be strengthened in such a way that it will become too strong a competitor in the product market. Preventing firms from collaborating in the product market may therefore destabilize $R \& D$ cooperatives, or prevent their formation in the first place. Our focus is on private incentives to develop cost saving technologies over time. In particular, we show that if firms collude in the product market, a wider range of technologies is fully developed. We also show that firms competing in the product market realize an inferior productive efficiency. We thus identify situations where product market collusion increases total surplus.

Dynamic models of R\&D were first introduced to study patent races whereby successful innovators capture the entire market. ${ }^{5}$ Meanwhile, a large literature has developed on the relation between intellectual property rights and antitrust policies. For instance, Quirmbach (1993) finds that there is an optimal level of collusion that is in between perfect competition and full collusion. And Green and Scotchmer (1995) show that it is optimal to allow for collusion through sequential licensing in case the next innovation is a truly new application of existing patents. More recently, another strand of dynamic R\&D models has developed: continuous-time generalizations of strategic R\&D models. ${ }^{6}$ Cellini and Lambertini (2005) is the first continuoustime generalization of the seminal analysis of d'Aspremont and Jacquemin (1988). In the duopoly game of d'Aspremont and Jacquemin (1988), firms first invest in cost-reducing R\&D and then play a Cournot game in the product market. In the continuous-time version of Cellini and Lambertini (2005), both firms start from an initial technology (that is, a level of marginal cost) and invest continuously in R\&D. This gradually reduces the initial level of marginal cost towards the steady-state level. In contrast to the static generalization of d'Aspremont and Jacquemin (1988) by Hinloopen (2000), Cellini and Lambertini (2005) find that the aggregate level of R\&D is monotonically increasing in the number of independent competitors.

We also consider a continuous-time generalization of d'Aspremont and Jacquemin (1988). There are two distinguishing features of our analysis. First, we consider all possible initial marginal cost levels, including those exceeding the choke price (the lowest price for which there is no demand). Especially in the early stages of development, it is quite likely that the cost of a new technology (the cost, say, to develop a prototype) exceeds the highest willingness to pay in the market. We characterize situations where such initial technologies are only developed if firms collude in the product market. Indeed, excluding initial marginal costs that are above the choke price ignores "... new ... processes ... [that] failed to come into existence, [as they are] killed by the Sherman Act before they were born." These instances constitute a direct welfare gain of product market collusion.

Second, in addition to near-equilibrium paths, we consider all trajectories that are candidates

\footnotetext{
${ }^{5}$ This literature starts with Loury (1979) and Lee and Wilde (1980); Reinganum (1989) surveys the early contributions. Patent race models examine, in essence, the time it takes for a cost-saving innovation to be completed. R\&D investments reduce this completion period. Because in these models the R\&D process itself cannot fail, the $\mathrm{R} \& \mathrm{D}$-investment decision is transformed into a static one.

${ }^{6}$ There is also a small literature that considers (stationary) repeated game models of R\&D; see Cabral (2000) and Lambertini et al. (2002). These 'dynamic' models do not allow for "smoothing the investment efforts over a long time" (Cellini and Lambertini, 2005), a type of investment behavior that is observed in practice and that constitutes a key feature of continuous-time models.
} 
for an optimal solution. This global analysis yields a bifurcation diagram that indicates for every possible parameter combination the qualitative features of any market equilibrium as well as of the transient dynamics towards it. ${ }^{7}$ We thus identify critical parameter values: points in parameter space at which the optimal investment function changes qualitatively. In particular, we determine the value of marginal costs for which $R \& D$ investments are terminated, and for which they are not initiated at all. We prove that these critical cost levels are affected by firm conduct. Therefore, extending the $R \& D$ cooperative to product market collusion can lead to qualitatively different long-run solutions, in spite of starting from an identical initial technology.

The related literature has not considered initial marginal cost levels that exceed the choke price, nor has it carried out a global analysis. ${ }^{8}$ The only exception is Hinloopen et al. (2013), who characterize the equilibria of a continuous-time dynamic monopoly with R\&D investments. We expand their analysis in three directions. First, we consider a duopoly rather than a monopoly. Second, we examine two different scenarios: one in which firms cooperate in R\&D and compete in the product market (labeled 'partial collusion') ${ }^{9}$, and one in which firms cooperate both in $\mathrm{R} \& \mathrm{D}$ and in setting price (labeled 'full collusion'). Indeed, comparing the two scenarios allows us to examine the effects of extending cooperation in $R \& D$ towards collusion in the product market. And third, rather than relying on numerical simulations, we prove a set of propositions that characterize the dynamics of the model throughout the entire parameter space.

Our framework yields four possible outcomes for any initial draw of a new technology (cf. Hinloopen et al., 2013). First of all, a 'promising technology' arrives, whereby the initial technology is developed through continuous R\&D investments. This can occur for initial cost levels both below and above the choke price. In the latter case, production starts only after some time, because early R\&D efforts have to bring down marginal cost below the choke price. Second, a 'strained market' arises: initial marginal cost is below the choke price and firms invest in $R \& D$, but the technology is not likely to be developed to full materialization. ${ }^{10}$ In case of an 'uncertain future', the third situation, it is not immediately clear whether the long-run steady state will be reached, or that it is optimal to gradually leave the market. Only time will tell. Fourth, an 'obsolete technology' can emerge: whatever the initial marginal cost, the technology is either not developed, or developed only to be taken off the market in due time. The long-run steady state will not be reached in either case.

All four technologies can emerge under both partial collusion and full collusion. Comparing

\footnotetext{
${ }^{7}$ Solution structures may change qualitatively due to variations in parameter values (indifference points may appear, steady states may lose their stability, and so on). These qualitative changes due to smooth variations in parameters are called bifurcations. For an introduction, see Grass et al. (2008), or Kiseleva and Wagener (2010).

${ }^{8}$ That literature is still small. It includes Cellini and Lambertini (2005, 2009), Lambertini and Mantovani (2009, 2010), and Kovac et al. (2010). In all these papers any of the initial (permissible) technologies will be developed to full materialization; technologies that are only developed under specific regimes (i.e. product market collusion) remain hidden.

${ }^{9}$ This scenario is also often called 'semi-collusion' in the literature.

${ }^{10}$ This situation resembles the 'sailing ship effect' of Cooper and Schendel (1976) (see also Howells, 2002), whereby the arrival of a new, possibly superior technology spurs the development of the old technology. In our case, there is no rival technology that induces continued investment in a technology that is bound to leave the market. Rather, it is the technology itself (characterized by the size of the initial marginal cost) that makes it optimal for firms to gradually take it off the market in due time.
} 
the two scenarios throughout the entire parameter space, we find that if firms collude in the product market (i) it is more likely that an initial technology qualifies as a Promising Technology, and if so, that it is more likely to be developed further, (ii) it is less likely that an initial technology qualifies as an Obsolete Technology, and if so, it is more likely that firms invest in $R \& D$, albeit temporarily, and (iii) if an initial technology causes a Strained Market or if it induces an Uncertain Future, it is less likely that it will be taken off the market in due time. Put differently, due to product market collusion it is more likely that firms invest in $\mathrm{R} \& \mathrm{D}$, and that these investments eventually lead to a steady state with positive production.

Our analysis qualifies the per se prohibition of collusion in product markets for high-tech industries. A higher total surplus obtains if colluding firms develop an initial technology and arrive at the saddle-point steady state while firms that compete in the product market would not develop the technology at all. We show that this is more likely to happen if new technologies arrive in circumstances that offer a high profit potential (that is, large markets and efficient R\&D processes). Under these circumstances, product market collusion can also yield higher total surplus if competing firms would develop the new technology as well, be it to take it off the market in due time, or to arrive at the saddle-point steady state. And in so far higher R\&D investments as such are desirable (as suggested in the endogenous growth literature; see e.g. Grossman and Helpman, 1991; Aghion and Howitt, 1992) the case for prohibiting collusion per $s e$ is further weakened. On the other hand, colluding firms tend to hold on longer to technologies that are destined to leave the market. This is not desirable from a social welfare point of view if that prevents the development of new, superior technologies.

A particularly difficult situation arises when the initial technology is above the choke price and if it will be developed only if firms collude in the product market. The welfare cost of prohibiting firms to collude then remains hidden because no production is affected by this prohibition. There is no production yet, and because collusion is prohibited, there will be no production in the future. Put differently, no production will be taken off the market if firms are prohibited to collude in the product market, leaving the welfare cost unnoticed. Our analysis thus offers a first glance at "new ... processes ... [that] failed to come into existence, killed by the Sherman Act before they were born."

The remainder of the paper is organized as follows. The basics of the model are introduced in Section 2. In Section 3, the necessary conditions for optimal production and investment schedules are derived under partial collusion and full collusion. The corresponding bifurcation diagrams are derived in Section 4 and the two scenarios are compared in Section 5. Section 6 concludes. An appendix contains the proofs of all propositions.

\section{The model}

Our present model is an extension of the global monopoly framework of Hinloopen et al. (2013) to two firms and builds on Cellini and Lambertini (2009). Time $t$ is continuous: $t \in[0, \infty)$. There are two a priori fully symmetric firms that both produce a homogeneous good at constant 
marginal costs $c_{i}(t)$. At every instant, the market price $p(t)$ is given as

$$
p(t)=A-Q(t)
$$

where $Q(t)=q_{1}(t)+q_{2}(t)$, with $q_{i}(t)$ the quantity produced by firm $i$ at time $t$, and where $A$ is the choke price. ${ }^{11}$

Each firm $i$ can reduce its marginal cost $c_{i}(t)$ by investing in R\&D. In particular, when firm $i$ exerts R\&D effort $k_{i}(t)$, its marginal cost evolves as

$$
\frac{d c_{i}}{d t}(t) \equiv \dot{c}_{i}(t)=c_{i}(t)\left(-k_{i}(t)-\beta k_{j}(t)+\delta\right),
$$

where $k_{j}(t)$ is the $R \& D$ effort exerted by its rival and where $\beta \in[0,1]$ measures the degree of spillover. Note that efficiency of production is assumed to decrease at a constant rate, as captured by $\delta>0$. This depreciation is due to (exogenous) aging of technology and organizational forgetting (Besanko et al. (2010), Lambertini and Mantovani, 2009). As Benkard (2004, p. 590) observes: “. . . an aircraft producer's stock of production experience is constantly being eroded by turnover, lay offs and simple losses of proficiency at seldom repeated tasks. When producers cut back output, this erosion can even outpace learning, causing the stock of experience to decrease." In our model, R\&D investment yields know-how gains but the logic of the argument is the same. For instance, complementary inputs that are typically purchased also constitute a fraction of production cost. Incorporating these inputs becomes ever more costly due to their inherent evolution over time, especially for firms that are relatively sluggish in $R \& D$, as $R \& D$ efforts also determine any firm's 'absorptive capacity' (Cohen and Levinthal, 1989). ${ }^{12}$

Both firms are endowed with an identical initial technology $c_{i}(0)=c_{j}(0)=c_{0}$, which is assumed to be drawn by Nature. Per unit of time, the costs of R\&D efforts are

$$
\Gamma_{i}\left(k_{i}\right)=b k_{i}^{2}
$$

where $b>0$ is inversely related to the cost-efficiency of the R\&D process. The R\&D process is thus assumed to exhibit decreasing returns to scale (Schwartzman, 1976; see also the discussion in Hinloopen et al., 2013). Both firms discount the future with the same constant rate $\rho>0$. Either firm's instantaneous profit therefore equals

$$
\pi_{i}\left(q_{i}, Q, k_{i}, c_{i}\right)=\left(A-Q-c_{i}\right) q_{i}-b k_{i}^{2},
$$

\footnotetext{
${ }^{11}$ We thus assume that the market size, $A$, is fixed and known to both firms. A random market size would not change any of our results qualitatively. See Hinloopen et al. (2013) for an analysis of unexpected changes in $A$.

${ }^{12} \mathrm{~A}$ non-positive depreciation rate yields trivial equilibria. Every initial technology will be developed in case $\delta$ is negative, as there is an exogenous reduction in marginal cost over time. For $\delta=0$, consider $\delta$ to be marginally positive. In that case, the value of initial marginal cost that would make it optimal not to invest in $R \& D$ is far above the choke price because only an infinitesimally small investment in R\&D is then needed to reduce marginal cost over time.
} 
with corresponding total discounted profit

$$
\Pi_{i}\left(q_{i}, Q, k_{i}, c_{i}\right)=\int_{0}^{\infty} \pi_{i}\left(q_{i}, Q, k_{i}, c_{i}\right) e^{-\rho t} d t
$$

The model has five parameters: $A, \beta, b, \delta$, and $\rho$. To simplify the analysis, we rescale the model such that it has only three parameters. Rescaling is done by choosing 'natural units' for the problem; it does not involve making special parameter choices. Rather, each choice of parameters in the original model corresponds to a choice of parameters in the rescaled model. The complexity reduction obtained by the scaling is a consequence of the fact that in the original parameters, many choices give rise to mathematically equivalent models. ${ }^{13,14}$

Lemma 1. By choosing the units of $t, q_{i}, q_{j}, c_{i}, c_{j}, k_{i}$, and $k_{j}$ appropriately, we can assume $A=1, b=1$, and $\delta=1$. This yields the following rescaled version of the model:

$$
\begin{gathered}
\tilde{\pi}_{i}\left(\tilde{q}_{i}, Q, \tilde{k}_{i}, \tilde{c}_{i}\right)=\left(1-\tilde{Q}-\tilde{c}_{i}\right) \tilde{q}_{i}-\tilde{k}_{i}^{2}, \\
\tilde{\Pi}_{i}\left(\tilde{q}_{i}, \tilde{Q}, \tilde{k}_{i}, \tilde{c}_{i}\right)=\int_{0}^{\infty} \tilde{\pi}_{i}\left(\tilde{q}_{i}, \tilde{Q}, \tilde{k}_{i}, \tilde{c}_{i}\right) e^{-\tilde{\rho} \tilde{t}} d \tilde{t} \\
\dot{\tilde{c}}_{i}=\tilde{c}_{i}\left(1-\left(\tilde{k}_{i}+\beta \tilde{k}_{j}\right) \phi\right), \quad \tilde{c}_{i}(0)=\tilde{c}_{0}, \quad \tilde{c}_{i} \in[0, \infty) \forall \tilde{t} \in[0, \infty) \\
\tilde{q}_{i} \geq 0, \quad \tilde{k}_{i} \geq 0 \\
\tilde{\rho}>0, \quad \phi>0
\end{gathered}
$$

with conversion rules: $q_{i}=A \tilde{q}_{i}, q_{j}=A \tilde{q}_{j}, k_{i}=\frac{A}{\sqrt{b}} \tilde{k}_{i}, k_{j}=\frac{A}{\sqrt{b}} \tilde{k}_{j}, c_{i}=A \tilde{c}_{i}, c_{j}=A \tilde{c}_{j}$, $\pi_{i}=A^{2} \tilde{\pi}_{i}, \pi_{j}=A^{2} \tilde{\pi}_{j}, \phi=\frac{A}{\delta \sqrt{b}}, t=\frac{\tilde{t}}{\delta}, \tilde{\rho}=\frac{\rho}{\delta}$.

Rescaling the model as in Lemma 1 introduces a new parameter: $\phi$. It is one-to-one related to the profit potential of a technology. Higher potential revenues come with a higher $A$, and each unit of R\&D effort costs more if $b$ increases, while it reduces marginal cost by less the higher is $\delta$. In sum, a lower (higher) $\phi$ corresponds to a lower (higher) profit potential. For notational convenience we henceforth omit tildes.

\section{Partial Collusion and Full Collusion}

In this section we derive the necessary conditions for optimal production and investment schedules in case firms cooperate in R\&D but compete in the product market (Section 3.1), and in case firms cooperate in $R \& D$ and collude in the product market (Section 3.2).

\footnotetext{
${ }^{13}$ To illustrate the usefulness of Lemma 1 , consider two models with different original parameterizations: i) $A=10$, $b=1, \delta=0.2, \rho=0.1, \beta=0.5$, ii) $A=20, b=4, \delta=0.2, \rho=0.1, \beta=0.5$. Both models correspond to the same rescaled model with $\phi=A / \delta \sqrt{b}=50, \tilde{\rho}=\rho / \delta=0.5, \beta=0.5$, and are therefore mathematically equivalent in the sense that they have the same solution in rescaled variables.

${ }^{14}$ The proof of Lemma 1 is analogous to the proof of Lemma 1 in Hinloopen et al. (2013).
} 


\subsection{Partial Collusion}

Both firms operate their own R\&D laboratory and production facility. They select their output levels non-cooperatively and adopt a strictly cooperative behavior in determining their R\&D efforts so as to maximize joint profits. These assumptions amount to imposing a priori the symmetry condition $k_{i}(t)=k_{j}(t)=k(t) .{ }^{15} c_{i}(0)=c_{j}(0)=c_{0}$ implies that $c_{i}(t)=c_{j}(t)=$ $c(t)$. Equation (8) thus reads as

$$
\dot{c}=c(1-(1+\beta) \phi k) .
$$

It may seem reasonable to assume that when firms cooperate in $R \& D$, they also fully share information, that is, to assume the level of spillover to be at its maximum $(\beta=1$; see Kamien et al., 1992). For the sake of generality, we do not a priori fix the value of $\beta$ at its maximal value. There are also intuitive arguments for not doing so as there might still be some ex post duplication and/or substitutability in $R \& D$ outputs if firms operate separate laboratories (see the discussion in Hinloopen, 2003).

The instantaneous profit of firm $i$ is

$$
\pi_{i}\left(q_{i}, Q, k, c\right)=(1-Q-c) q_{i}-k^{2}
$$

with $Q=q_{1}+q_{2}$, yielding its total discounted profit over time

$$
\Pi_{i}\left(q_{i}, Q, k, c\right)=\int_{0}^{\infty} \pi_{i}\left(q_{i}, Q, k, c\right) e^{-\rho t} d t .
$$

As firms jointly decide on their R\&D efforts, the only independent decisions are those of production. However, as quantity variables do not appear in the equation for the state variable (11), production feedback strategies of a dynamic game are simply static Cournot-Nash strategies of each corresponding instantaneous game.

Maximizing $\pi_{i}$ over $q_{i} \geq 0$ gives us standard Cournot best-response functions for the product market

$$
q_{i}\left(q_{j}\right)=\left\{\begin{array}{ccc}
\frac{1}{2}\left(1-c-q_{j}\right) & \text { if } & q_{j}<1-c \\
0 & \text { if } & q_{j} \geq 1-c .
\end{array}\right.
$$

Note that the constraint $q_{i} \geq 0$ is binding when $q_{j} \geq 1-c$. Solving for Cournot-Nash production levels, we obtain

$$
q^{N}=\left\{\begin{array}{ccc}
\frac{1}{3}(1-c) & \text { if } & c<1 \\
0 & \text { if } & c \geq 1 .
\end{array}\right.
$$

\footnotetext{
${ }^{15}$ Throughout the paper we consider symmetric equilibria only. See Salant and Shaffer (1998) for a specific example of a static model of R\&D where individual firms face different capacity constraints in which it is optimal for firms in an $R \& D$ cooperative to make unequal investments.
} 
Consequently, the instantaneous profit of each firm is ${ }^{16}$

$$
\pi(c, k)=\left\{\begin{array}{r}
\frac{1}{9}(1-c)^{2}-k^{2} \quad \text { if } \quad c<1 \\
-k^{2} \quad \text { if } \quad c \geq 1
\end{array}\right.
$$

The dynamic optimization problem of the $R \& D$ cooperative boils down to finding an $R \& D$ effort schedule $k^{*}$ for either firm that maximizes the total discounted joint profit, taking into account the state equation (11), the initial condition $c(0)=c_{0}$, and the control constraint $k(t) \geq 0$ which must hold at all times. Note that according to (11), if $c_{0}>0$, then $c(t)>0$ for all $t$. The state space of this problem is the interval $[0, \infty)$ of marginal cost levels.

To solve this problem, we introduce the current-value Pontryagin function (also called the un-maximized Hamilton or pre-Hamilton function) ${ }^{17}$

$$
P(c, k, \lambda)=\left\{\begin{array}{rll}
\frac{1}{9}(1-c)^{2}-k^{2}+\lambda c(1-(1+\beta) \phi k) & \text { if } & c<1 \\
-k^{2}+\lambda c(1-(1+\beta) \phi k) & \text { if } & c \geq 1
\end{array}\right.
$$

where $\lambda$ is the current-value co-state variable of a firm in the R\&D cooperative. The co-state (or shadow value) measures the marginal worth of the increment in the state $c$ for each firm at time $t$ when moving along the optimal path. We expect $\lambda(t) \leq 0$ along optimal trajectories because marginal cost is a "bad".

We use Pontryagin's maximum principle to obtain the solution to our optimization problem. Maximizing over the control $k \geq 0$ yields

$$
k=\max \left\{0,-\frac{1}{2} \lambda c(1+\beta) \phi\right\} .
$$

The maximum principle states further that the optimizing trajectory necessarily corresponds to the trajectory of the state-costate system

$$
\dot{c}=\frac{\partial P}{\partial \lambda}, \quad \dot{\lambda}=\rho \lambda-\frac{\partial P}{\partial c},
$$

where $k$ is replaced by its maximizing value. For $\lambda \leq 0$, relation (18) gives a one-to-one correspondence between the co-state $\lambda$ and the control $k$. We use this relation to transform the state-costate system into a state-control system which an optimizing trajectory has to satisfy necessarily as well. This system consists of two regimes (following the two part composition

\footnotetext{
${ }^{16}$ We implicitly assume that firms face no financial constraints; they can invest in R\&D prior to production. Credit rationing would impose an upper limit on the value of an indifference point; qualitatively it would not change our conclusions. For a sample of Italian manufacturing firms Piga and Atzeni (2007) find that credit constraints are negligible for R\&D intensive firms. Bond et al. (2005) find no significant relationship between the level of R\&D investments and cash flow for German and U.K firms, while Harhoff (1998) finds a weak but statistically significant relationship for both small and large German firms. The sensitivity of R\&D investments to cash flow fluctuations seems to be stronger for U.S. firms (e.g., Himmelberg and Petersen (1994), Hall et al. (1999)), but by and large, the literature on the importance of financial constraints for R\&D investment is inconclusive (see Hall and Lerner (2010) for an overview).

${ }^{17} \mathrm{We}$ omit a factor 2 for joint profits to obtain the solution expressed in per-firm values. Due to symmetry, maximizing per-firm total profit corresponds to maximizing joint total profit.
} 
of the Pontryagin function). The first one corresponds to $c<1$ and positive production ( $q=$ $(1-c) / 3)$. The second one corresponds to $c \geq 1$ and zero production. ${ }^{18}$ The state-control system with positive production consists of the following two differential equations:

$$
\left\{\begin{array}{l}
\dot{c}=c(1-(1+\beta) \phi k), \\
\dot{k}=\rho k-\frac{(1+\beta) \phi}{9} c(1-c) .
\end{array}\right.
$$

The state-control system with zero production is given by

$$
\left\{\begin{array}{l}
\dot{c}=c(1-(1+\beta) \phi k), \\
\dot{k}=\rho k .
\end{array}\right.
$$

\subsection{Full Collusion}

Under full collusion, firms determine jointly their R\&D efforts and their output levels. This amounts to imposing a priori the symmetry conditions $k_{i}(t)=k_{j}(t)=k(t)$ and $q_{i}(t)=q_{j}(t)=$ $q(t)$. Equation (8) reads again as Equation (11). The profit of each firm at every instant is

$$
\pi(q, k, c)=(1-2 q-c) q-k^{2}
$$

with corresponding total discounted profit

$$
\Pi(q, k, c)=\int_{0}^{\infty} \pi(q, k, c) e^{-\rho t} d t .
$$

The optimal control problem of the two colluding firms is to find controls $q^{*}$ and $k^{*}$ that maximize the profit functional $\Pi$ subject to the state equation (11), the initial condition $c(0)=c_{0}$, and two control constraints that must hold at all times: $q \geq 0$ and $k \geq 0 .{ }^{19}$ Notice again that according to (11), if $c_{0}>0$, then $c(t)>0$ for all $t$.

The current-value Pontryagin function in case of full collusion reads as:

$$
P(c, q, k, \lambda)=(1-2 q-c) q-k^{2}+\lambda c(1-(1+\beta) \phi k)
$$

where $\lambda$ is the current-value co-state variable. It now measures the marginal worth at time $t$ of an increment in the state $c$ for a colluding firm when moving along the optimal path.

The necessary conditions for the solution to the dynamic optimization problem consist again of a state-control system which has two regimes. As in the partial collusion case, the first regime corresponds to $c<1$ and positive production $(q=(1-c) / 4)$, while the second regime corresponds to $c \geq 1$ and zero production.

\footnotetext{
${ }^{18}$ Recall from Lemma 1 that $A=1$ in the rescaled model. In the non-rescaled model, the analogous conditions for positive and zero production are $c(t)<A$ and $c(t) \geq A$, respectively.

${ }^{19}$ Again, due to symmetry, maximizing per-firm total profit corresponds to maximizing joint total profit.
} 
The state-control system in the region with positive production reads as

$$
\left\{\begin{array}{l}
\dot{c}=c(1-(1+\beta) \phi k), \\
\dot{k}=\rho k-\frac{(1+\beta) \phi}{8} c(1-c),
\end{array}\right.
$$

whereas the state-control system with zero production is

$$
\left\{\begin{array}{l}
\dot{c}=c(1-(1+\beta) \phi k), \\
\dot{k}=\rho k .
\end{array}\right.
$$

\section{Analysis}

Consider the system

$$
\left\{\begin{array}{l}
\dot{c}=c(1-(1+\beta) \phi k), \\
\dot{k}=\rho k-\alpha \phi(1+\beta) c(1-c) \chi(c),
\end{array}\right.
$$

where $\chi(c)=1$ if $0<c<1$ and $\chi(c)=0$ if $c \geq 1$ (or $c \leq 0$ ). Systems (20) - (21) and (25) - (26) are instances of system (27), with $\alpha=1 / 9$ for the partial collusion scenario and $\alpha=1 / 8$ for the full collusion scenario. ${ }^{20}$

The first result gives the properties of the steady states of the state-control system (see Appendix A.1 for the proof).

\section{Proposition 1. Let}

$$
D=\frac{1}{4}-\frac{\rho}{\alpha(1+\beta)^{2} \phi^{2}} .
$$

Depending on the value of $D$, there are three different situations.

1. If $D>0$, the state-control system with positive production (25) has three steady states:
i. $\left(c^{e}, k^{e}\right)=(0,0)$ is an unstable node,
ii. $\left(c^{e}, k^{e}\right)=\left(\frac{1}{2}+\sqrt{D}, \frac{1}{(1+\beta) \phi}\right)$ is either an unstable node or an unstable focus, and
iii. $\left(c^{e}, k^{e}\right)=\left(\frac{1}{2}-\sqrt{D}, \frac{1}{(1+\beta) \phi}\right)$ is a saddle-point steady state.

2. At $D=0$, there are two steady states:
i. $\left(c^{e}, k^{e}\right)=(0,0)$, which is an unstable node, and
ii. $\left(c^{e}, k^{e}\right)=\left(\frac{1}{2}, \frac{1}{(1+\beta) \phi}\right)$, which is a semi-stable steady state.

3. If $D<0$, the origin $\left(c^{e}, k^{e}\right)=(0,0)$ is the unique steady state of the state-control system with positive production, which is unstable.

The system consequently exhibits a saddle-node bifurcation at $D=0$.

\footnotetext{
${ }^{20}$ The monopoly system in Hinloopen et al. (2013) is also a special case of system (27), with $\alpha=1 / 4$.
} 
The stable manifold of the saddle-point steady state is one of the candidates for an optimal solution. However, as neither the Mangasarian nor the Arrow concavity conditions are satisfied, the stable manifold is not necessarily optimal. Note that Proposition 1 already implies that there should be other candidates for optimality as there is a parameter region for which there is no saddle point, and hence no stable manifold to it. The following result clarifies (Appendix A.2 contains the proof).

Proposition 2. The set of candidates for an optimal solution consists of the stable paths $W_{-}^{s}$ of the saddle-point steady state and the trajectory E through the point $(c, k)=(1,0)$.

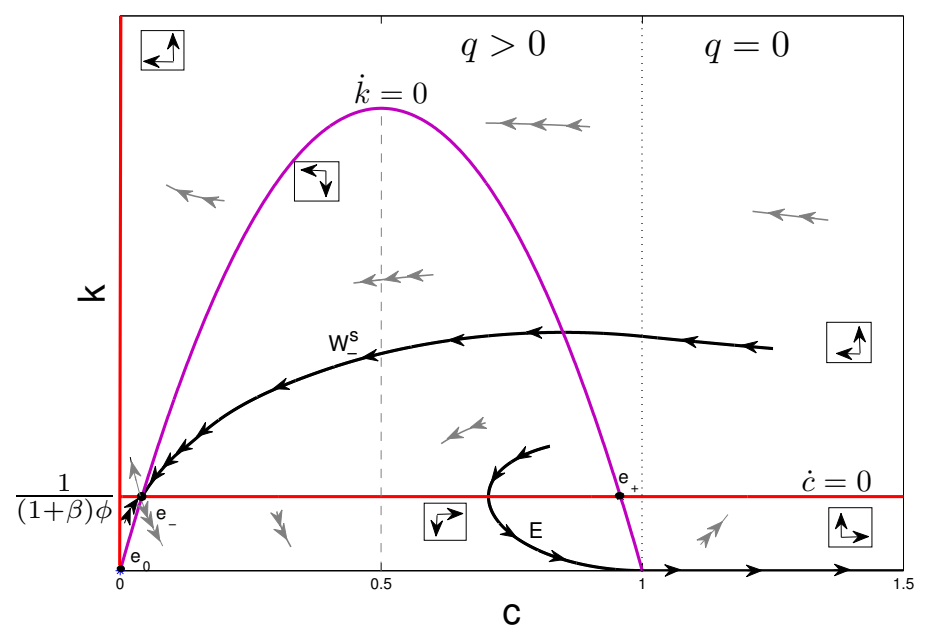

Figure 1: Candidate maximizing trajectories $W_{-}^{s}$ and $E$ in the state-control space.

Proposition 2 is illustrated in Figure 1. The thick black lines $W_{-}^{s}$ and $E$ indicate optimal solutions. The dotted vertical line $c=1$ separates the region with zero production from the region of positive production. We label the trajectory $E$ the "exit trajectory", as following this trajectory implies that firms eventually leave the region with positive production.

Proposition 2 only reduces the set of trajectories by applying necessary conditions for optimality, but there is no guarantee that an optimal solution exists. The next proposition summarizes when an optimal solution exists (the proof is in Appendix A.3).

Proposition 3. For all admissible values of the parameters, the following is true. At all initial points, the optimal control problem has at least one solution, which is among the candidates specified in Proposition 2. Moreover, there is at most one initial state $\hat{c}$ such that there are two optimizing trajectories starting at $\hat{c}$.

To assess the dependence of the solution structure on the model parameters, we carry out a bifurcation analysis. This consists of identifying those parameter values for which the qualitative structure of the optimal dynamics changes. These 'bifurcating' values bound open parameter regions such that the optimal dynamics are qualitatively identical for all parameter values in a region (see Wagener, 2003, Kiseleva and Wagener, 2015). Put differently, for all points in a 
region, a sufficiently small change in parameter values will not lead to a qualitative change of the optimal dynamics; regions characterize stable types of dynamics.

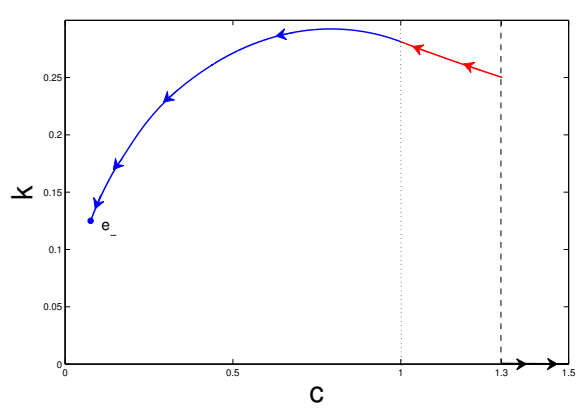

(a) Promising Technology

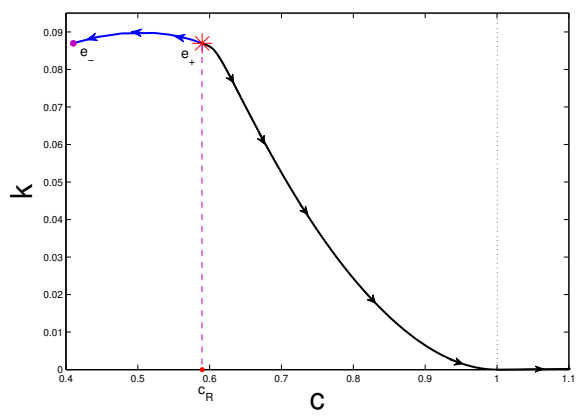

(c) Uncertain future

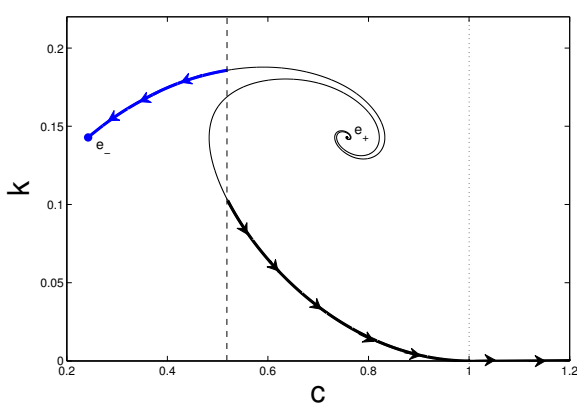

(b) Strained Market

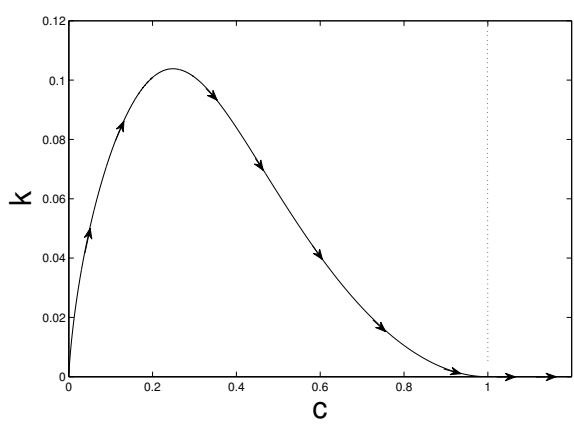

(d) Obsolete technology

Figure 2: R\&D investment trajectories for the four stable dynamics types of system (27).

System (27) has four distinct stable dynamics types (cf. Hinloopen et al., 2013). These are illustrated in Figure 2 in case of partial collusion. ${ }^{21}$ The first type is a "Promising Technology". In this case there exists an initial technology $\hat{c}>1$ that is an indifference threshold: ${ }^{22}$ a point in state space where the decision maker is indifferent between two optimal trajectories that have distinct long-term limit behavior. In particular, for $0<c_{0} \leq \hat{c}$ it is optimal to start developing the initial technology, ending up in the saddle-point steady state in the region of positive production. If $1<c_{0} \leq \hat{c}$, initially firms invest only in $\mathrm{R} \& \mathrm{D}$; production begins whenever $c(t)<1$. If $c_{0} \geq \hat{c}$, it is optimal not to initiate R\&D efforts; potential future profits do not suffice to compensate for losses that would be incurred in the initial periods during which firms would invest in R\&D but would not produce yet. ${ }^{23}$

The second type corresponds to a "Strained Market", where there is an indifference threshold below the choke price (that is, in the region with positive production): $0<\hat{c}<1$. In this case, if $0<c_{0}<\hat{c}$, the initial technology will be developed towards the saddle-point steady

\footnotetext{
${ }^{21}$ The same types emerge under full collusion. The stable dynamics types are compared across scenarios in Section 5 .

${ }^{22}$ Also known as Skiba, Dechert-Nishimura-Skiba or DNSS point; see Grass et al. (2008).

${ }^{23}$ Note that for $c_{0}=\hat{c}$, there are two distinct R\&D investment trajectories, which are, nevertheless, both optimal; see also Proposition 3.
} 
state. If $\hat{c}<c_{0}<1$, the exit-trajectory applies; $R \& D$ investments only serve to slow down the technological decay.

In a small part of the parameter space the third type arises: an "Uncertain Future". Initial technologies (states) are now divided by a repelling steady state (rather than an indifference point). If the system starts exactly at the repelling point, it stays there indefinitely; when it starts close to it, it stays there for a long period of time, after which it converges to one of the two attractors: the steady state or the exit trajectory.

The fourth type typifies the dynamics of an "Obsolete Technology". Whatever the initial technology, (eventually) the firms leave the market; R\&D investments are only used to slow down the technical decay.

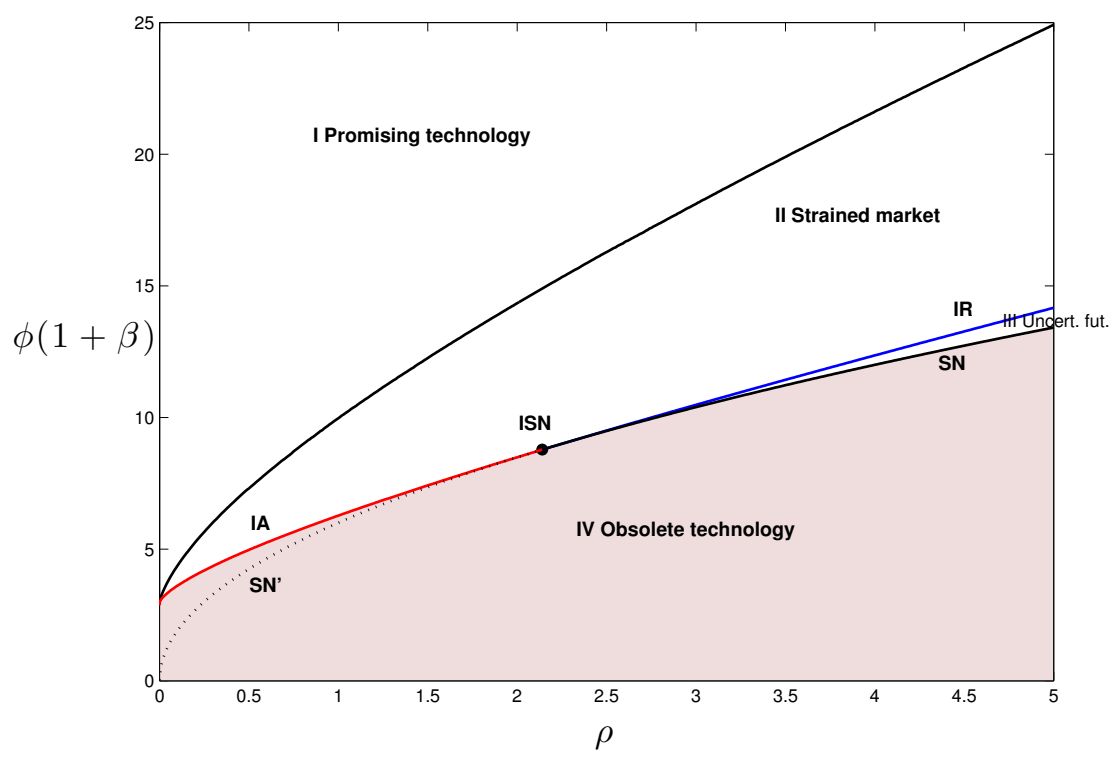

Figure 3: Bifurcation diagram (partial collusion).

The four different dynamics types are grouped conveniently in a bifurcation diagram (see Figure 3): the graph that indicates for every possible parameter combination the qualitative features of any market equilibrium as well as the transient dynamics towards them. In Figure 3, the uppermost curve represents parameter values for which the indifference point is exactly at $c=1$. At the saddle-node curve ( $\mathrm{SN})$, an optimal repeller and an optimal attractor collide and disappear. The curve SN' corresponds to saddle-node bifurcations in the state-control system that do not correspond to optimal dynamics. At the indifference-attractor bifurcations (IA), an indifference point collides with an optimal attractor and both disappear. Finally, at an indifference-repeller bifurcation (IR), an indifference point turns into an optimal repeller. The central indifference-saddle-node (ISN) bifurcation point at $(\rho, \phi(1+\beta)) \approx(2.14,8.78)$ organizes the bifurcation diagram. The curve representing indifference points at $c=1$ obtains a value of $\phi(1+\beta) \approx 2.998$ for $\rho=1 \times 10^{-5}$. 


\section{Collusion and the incentives to innovate}

In this section we compare the global optimum of the two scenarios. For a welfare comparison, we introduce total discounted values of profits (П), consumer surplus (CS), and total surplus (TS)

$$
\begin{gathered}
\Pi=\int_{0}^{\infty} \pi(t) e^{-\rho t} d t \\
C S=\int_{0}^{\infty} \frac{1}{2}(1-p(t)) Q(t) e^{-\rho t} d t=\int_{0}^{\infty} 2 q(t)^{2} e^{-\rho t} d t \\
T S=2 \Pi+C S,
\end{gathered}
$$

where at time $t=0$ firms start with $c_{0}$ and then invest along the optimal trajectory $\gamma(t)=$ $(c(t), k(t))$ as $t \rightarrow \infty$.

We first formally establish that the two scenarios yield different (optimal) trajectories. In Figure 4 the bifurcation diagrams of both scenarios are superimposed. There are significant quantitative differences between the two diagrams, as reflected by the different locations of the curves that divide the parameter space. Let $I_{i}, I I_{i}, \ldots, i=1,2$ denote regions $I, I I, \ldots$ under scenario $i$, with $i=1$ (2) corresponding to partial (full) collusion. The following then holds (see Appendix A.4 for the proof).

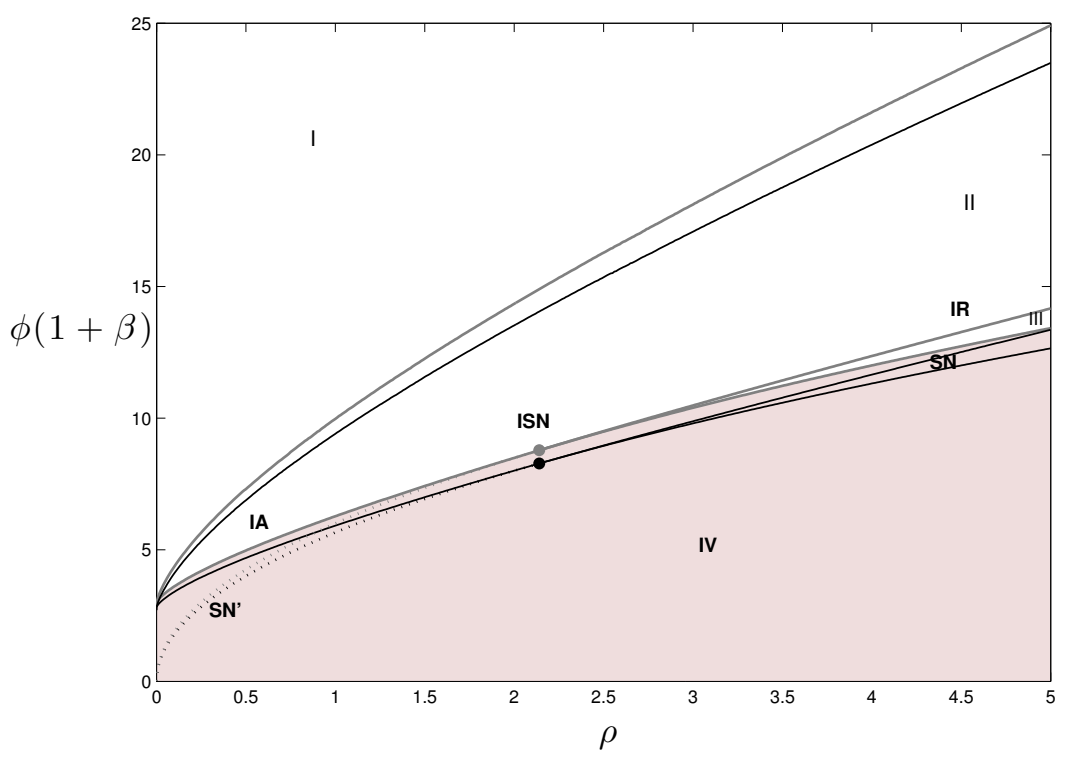

Figure 4: Superimposed bifurcation diagrams. Curves of the partial (full) collusion scenario are grey (black). 
Proposition 4. The following inclusions hold:

$$
\begin{aligned}
I_{1} & \subset I_{2}, \\
I_{1} \cup I I_{1} & \subset I_{2} \cup I I_{2}, \\
I_{1} \cup I I_{1} \cup I I I_{1} & \subset I_{2} \cup I I_{2} \cup I I I_{2} .
\end{aligned}
$$

The first inclusion of Proposition 4 implies that the "Promising Technology" region is larger if firms collude in the product market; due to collusion, the situation where firms first invest in $\mathrm{R} \& \mathrm{D}$, and only after some initial development period start producing, is more likely to occur. From the third inclusion follows that the "Obsolete Technology" region is smaller if firms collude; firms that collude are less likely either not to develop an initial technology, or to invest in R\&D only to abandon the technology in time.

\subsection{R\&D investment incentives}

In line with much of the related literature (Tirole, 1988), Proposition 4 suggests that colluding firms have in general a stronger incentive to invest in R\&D. This turns out to be the case, as the next proposition formally shows (see Appendix A.5.1 for the proof).

Proposition 5. Investment in $R \& D$ in the full collusion scenario is always at least as high as in the corresponding partial collusion scenario.

Proposition 5 implies the following. First of all, whenever both scenarios lead to the saddlepoint steady state, marginal costs in the full collusion scenario are lower than in case of partial collusion, because fully colluding firms have invested more in cost-reducing $R \& D$ to arrive at the long-run equilibrium. Put differently, product market collusion yields a higher production efficiency.

Second, if the initial technology leads to production after some initial development period only, colluding firms will enter this production phase more quickly because at every instant of the pre-production phase they invest more in R\&D in order to bring the level of marginal costs below the choke price.

Third, firms that collude in the product market abandon obsolete technologies at a lower pace. This implication has a similar vein as the argument of Arrow (1962), that a monopolist has less incentive to invest in R\&D than an otherwise identical but perfectly competitive market, because by doing so the monopolist replaces current monopoly profits by future (higher) monopoly profits. Here, the alternative for colluding firms is to exit the market more quickly (rather than staying in the market as a monopolist, as in Arrow, 1962), an alternative that for them is not optimal (see Figure 5).

The difference in R\&D intensity across the two scenarios is also reflected in the type of trajectories that firms select. To characterize this difference for all possible situations, it is convenient to have defined the threshold level of initial marginal cost $\hat{c}$ between 'eventual exit' 


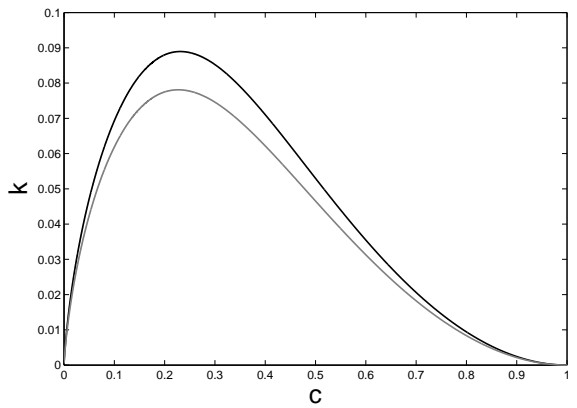

(a)

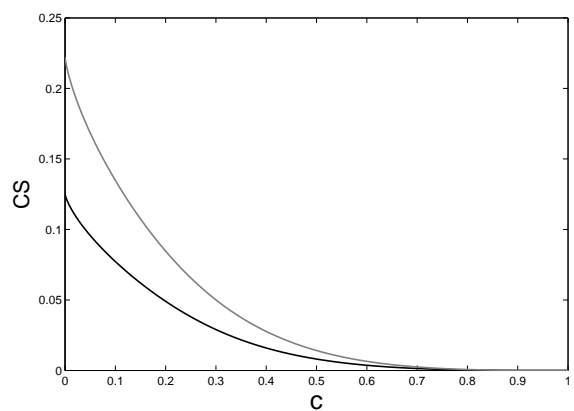

(c)

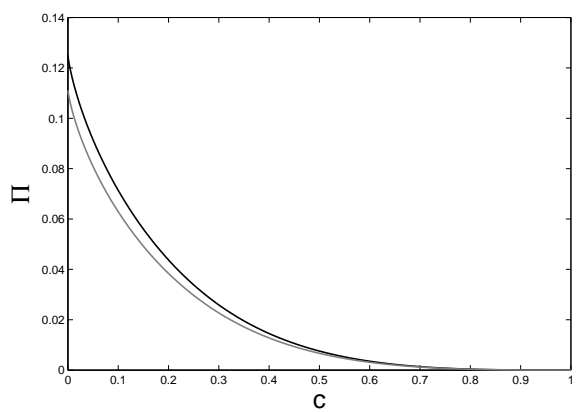

(b)

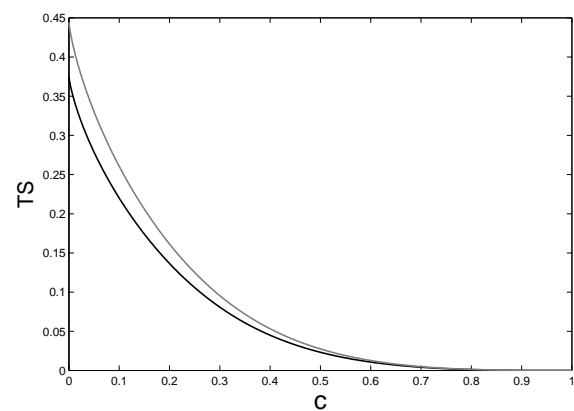

(d)

Figure 5: State-control space (a), total discounted profit (b), consumer surplus (c), and total surplus $(d)$, when the exit trajectory is an optimal solution. Parameters: $(\beta, \rho, \phi)=(1,1,2)$. Curves of the partial (full) collusion scenario are grey (black).

and 'eventual positive production'. Formally, set $\hat{c}=0$ in the "Obsolete Technology" region and let $\hat{c}_{1}$ and $\hat{c}_{2}$ denote the threshold level for the partial collusion and the full collusion scenarios, respectively. We can then state the following (see Appendix A.5.2 for the proof).

Proposition 6. For all parameter values, either $\hat{c}_{1}<\hat{c}_{2}$ or $\hat{c}_{1}=\hat{c}_{2}=0$.

The implications of Proposition 6 are twofold. First, if firms collude in the product market, the set of initial technologies that are developed towards the saddle-point steady state is larger (see Figure 6). In particular, if the initial technology $c_{0}$ falls in the non-empty interval $\left(\hat{c}_{1}, \hat{c}_{2}\right)$, it will only be brought to full materialization if firms collude in the product market.

Second, the set of initial technologies that triggers no investment in R\&D at all or that induces firms to select the exit trajectory is smaller if firms collude in the product market. Figure 7 illustrates this for a Strained Market. The strained investment circumstances induce partially colluding firms to exit the market in due time for all $c_{0}>\hat{c}_{1}$. In contrast, fully colluding firms exit the market only for $c_{0}>\hat{c}_{2}$. Initial technologies $c_{0}$ in the interval $\left(\hat{c}_{1}, \hat{c}_{2}\right)$ are therefore only brought to full maturation by firms that collude in the product market.

We can conclude that due to collusion in the product market (i) it is more likely that an initial technology qualifies as a Promising Technology, and if so, that it is more likely to be developed further, (ii) it is less likely that an initial technology qualifies as an Obsolete Technology, and if 


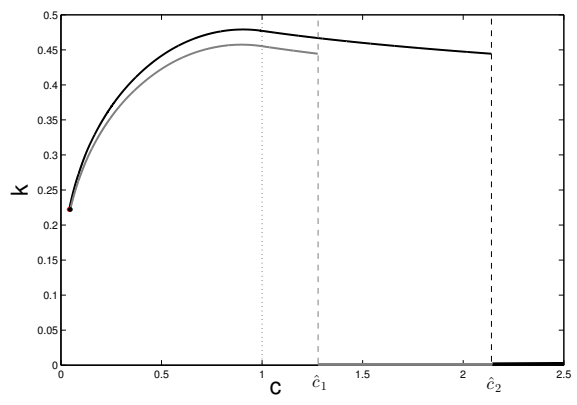

(a)

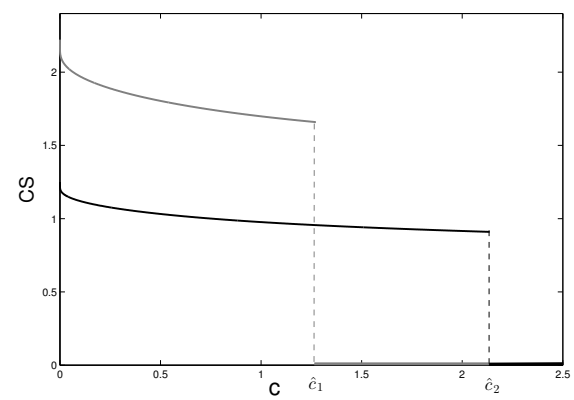

(c)

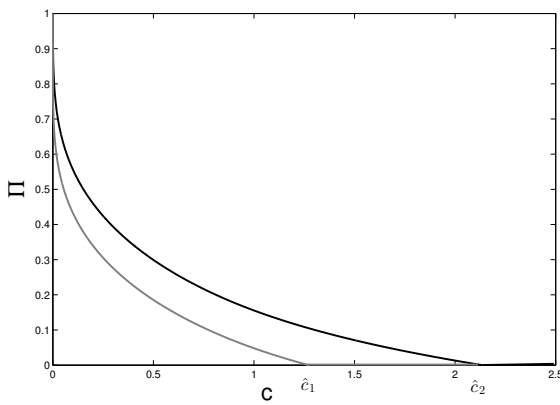

(b)

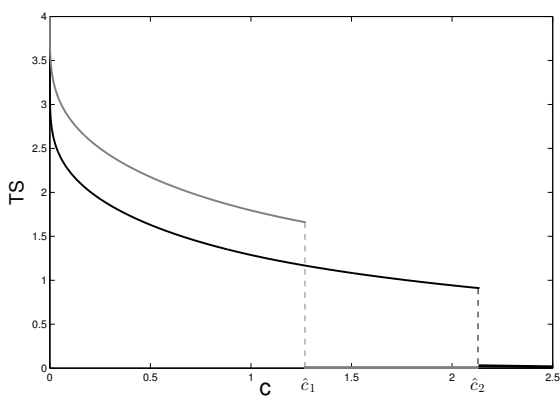

(d)

Figure 6: State-control space (a), total discounted profit (b), consumer surplus (c), and total surplus $(d)$, when the indifference point is in the region with zero production. Parameters: $(\beta, \rho, \phi)=(1,0.1,2.25)$. Curves of the partial (full) collusion scenario are grey (black).

so, it is more likely that firms invest in R\&D, albeit temporarily, and (iii) if an initial technology causes a Strained Market or if it induces an Uncertain Future, it is less likely that it will be taken off the market in due time. Put differently, due to product market collusion it is more likely that firms invest in R\&D, and that these investments eventually lead to a steady state with positive production.

\subsection{Total surplus}

We next consider the effect of product market collusion on total surplus. Obviously, collusion in the product market yields higher total surplus if colluding firms develop an initial technology and arrive at the saddle-point steady state while firms that compete in the product market would not develop the technology at all. Formally, ${ }^{24}$

Proposition 7. Whenever both scenarios have an indifference point above the choke price, the full collusion scenario yields higher consumer surplus and total surplus than the partial collusion scenario for all initial technologies in between the two indifference points.

\footnotetext{
${ }^{24}$ The proof of Proposition 7 follows trivially from the fact that $i$ ) for all values of $c$ above the indifference point in the region where $c \geq 1$, both $q=0$ and $k=0$ for all $t \in[0, \infty)$, and $i i)$ for all values of $c$ below the indifference point, $\Pi>0$ and for some finite time $T$ also $q>0$ for all $t>T$ as $t \rightarrow \infty$.
} 


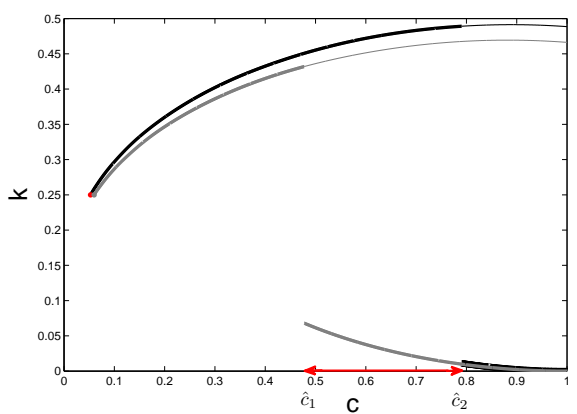

(a)

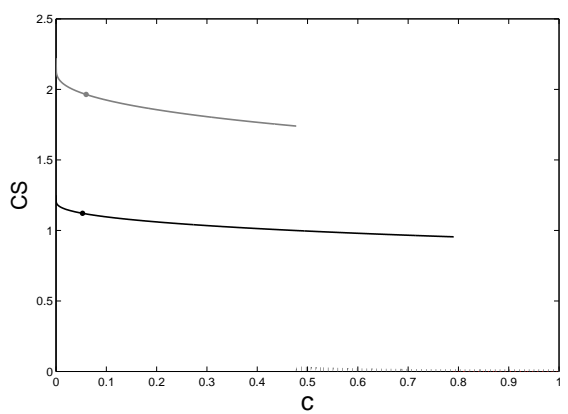

(c)

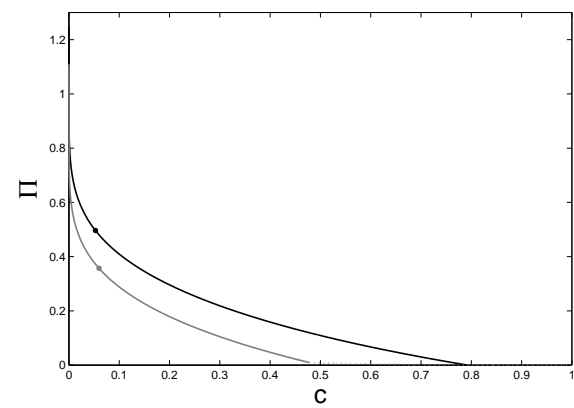

(b)

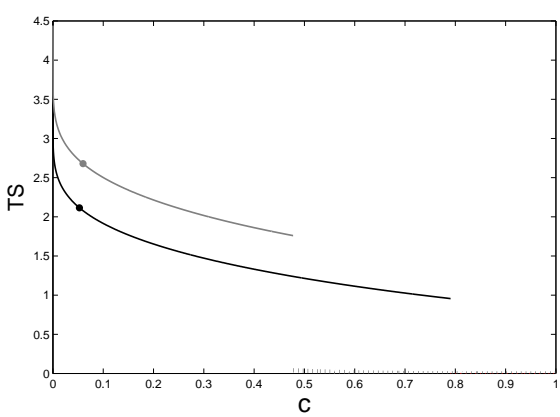

(d)

Figure 7: State-control space (a), total discounted profit (b), consumer surplus (c), and total surplus $(d)$, when the indifference point is within the region with positive production. Parameters: $(\beta, \rho, \phi)=(1,0.1,2)$. Curves of the partial (full) collusion scenario are grey (black); curves of the stable path (exit trajectory) are solid (dotted). Dots indicate the saddle-point steady state.

Figure 6 illustrates Proposition 7: for all $c_{0} \in\left(\hat{c}_{1}, \hat{c}_{2}\right)$, collusion in the product market yields a higher total surplus. Figure 8 illustrates some comparative statics of the indifference points in this case. Indeed, these points are positively related to market size and R\&D efficiency. Note, however, that also $\Delta \hat{c}=\hat{c}_{2}-\hat{c}_{1}$ increases if the $\mathrm{R} \& \mathrm{D}$ process becomes more efficient and/or if the market size becomes larger, the more so the lower is the discount rate (in Figure 8, a lower discount rate corresponds to a larger slope of the convex curves). Because future mark-ups are positively related to both market size and R\&D efficiency, an increase in either of these two has a larger (positive) effect on future profits if firms collude in the product market. And these future benefits feature more prominently in total discounted profits if the discount rate is lower. Therefore, indifference points correspond to lower marginal costs values if the discount rate goes up, all else equal (cf. the relative location of $C_{1}$ and $C_{2}$ in Figure 8).

Product market collusion can also yield higher total surplus if colluding firms arrive at the saddle-point steady state while firms that compete in the product market would select the exit trajectory. In these cases, firms that compete in the product market temporarily produce more. This is off-set by the added benefits of sustained R\&D investments under full collusion if the discount rate is sufficiently small (see Figure 7).

Finally, collusion in the product market can also yield a higher total surplus if under both 


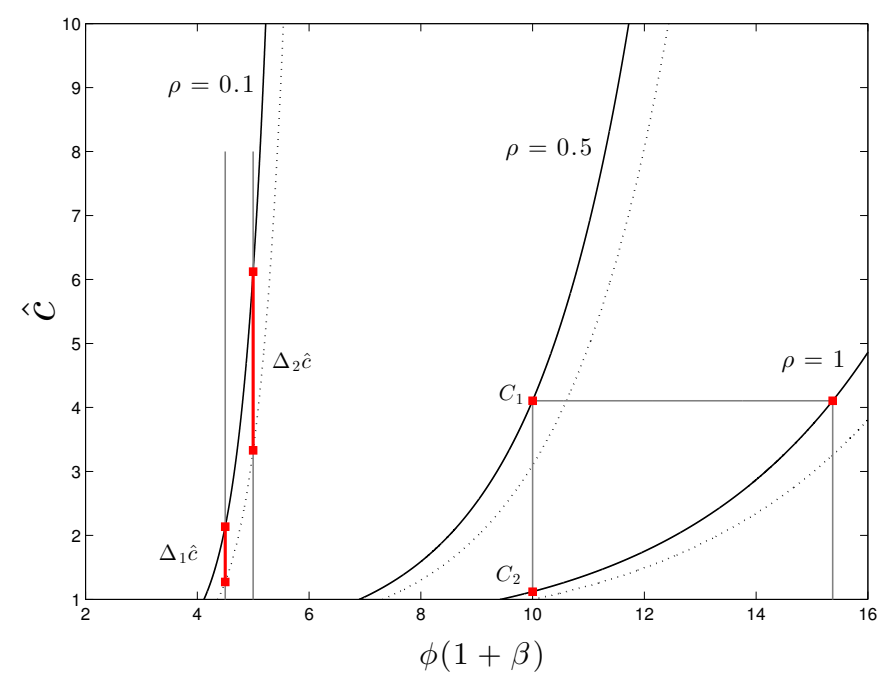

Figure 8: Dependence of the indifference point $\hat{c}$ on model parameters. Curves of the partial (full) collusion scenario are dotted (solid).

scenarios firms would select the trajectory towards the saddle-point steady state: in Figure 9, for all $c_{0} \in\left(c^{\star}, \hat{c}_{2}\right)$, total surplus is higher if firms collude in the product market. In this example, the discount rate is high: $\rho=10$, which corresponds, for instance, to the non-rescaled variables $\delta=0.01$ and $\rho=0.1$. Also, the initial marginal costs are sufficiently high. In such an environment, the higher $R \& D$ investments and the reduced importance that is attached to future surplus work in favor of product market collusion as under this scenario firms will reach the production stage more quickly, a benefit that more than off-sets the welfare loss of future increased mark-ups. ${ }^{25}$

\section{Concluding remarks}

Schumpeter's famous observation continues to challenge the design of optimal competition policies for high tech sectors. The classic rationale for competition policies is rooted in their effect on total surplus. Typically, product market collusion transfers consumer surplus to firm profits, resulting in a net loss of total surplus. To date, the literature considers this result to be robust to the increased incentive to invest in $R \& D$ that comes with collusion in the product market. Our analysis shows that it actually fails this robustness check if the phase of development prior to production is taken into account and/or if all possible $R \& D$ investment trajectories are considered.

According to our analysis, extending an $R \& D$ cooperative agreement to collusion in the product market is welfare enhancing if the market size is large and/or the R\&D process is efficient, given a relatively modest discount rate. The profit potential of a new technology is then relatively

\footnotetext{
${ }^{25}$ More precisely, a higher (rescaled) discount rate $\tilde{\rho}=\rho / \delta$ implies either a higher discount rate $\rho$ or a lower $\delta$. With a lower $\delta$, cost reductions take longer, such that the time difference in reaching the production stage between the two scenarios becomes more pronounced.
} 


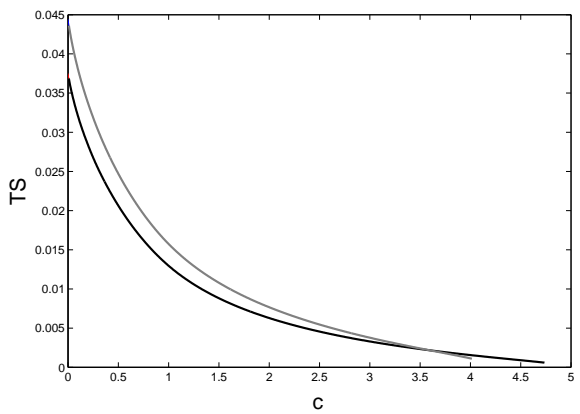

(a)

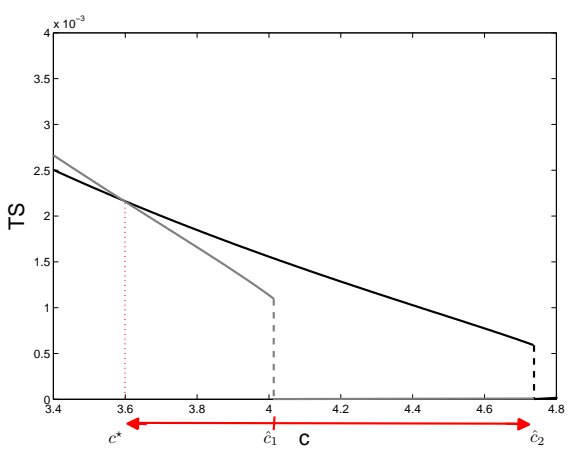

(b)

Figure 9: Total surplus when the indifference point is in the region with zero production. Parameters: $(\beta, \rho, \phi)=(1,10,50)$. Grey curves correspond to partial collusion, whereas the black ones correspond to full collusion. $c^{\star} \approx 3.6, \hat{c}_{1} \approx 4.01, \hat{c}_{2} \approx 4.74$. For all $c_{0} \in\left(c^{\star}, \hat{c}_{2}\right)$, total surplus is higher if firms collude in the product market.

large. As a result, firms that collude in the product market bring more initial technologies to full materialization.

A particularly disturbing situation arises when the initial draw $c_{0}$ out of $\left(\hat{c}_{1}, \hat{c}_{2}\right)$ is above the choke price $\left(c_{0}>1\right)$. The welfare cost of prohibiting firms to collude in the product market then remains hidden because no production is affected by this prohibition. There is no production yet, and because collusion is prohibited, there will be no production in the future. Put differently, no production will be taken off the market if firms are prohibited to collude in the product market.

Our analysis thus signals a potential problem for antitrust policy as it shows that prohibiting collusion in the product market per se is not univocally welfare enhancing. It also shows that the associated welfare costs might not surface.

By enabling firms to smooth their investments over time, the continuous-time approach to modeling strategic R\&D, introduced by Cellini and Lambertini $(2005,2009)$, brought theory closer to reality. Hinloopen et al. (2013) however pointed out that in these models sufficient optimality conditions were not satisfied and numerically established a possibility of a technology exiting the market or never being developed in the first place; a possibility of investments preceding production was also introduced for the first time. This resulted in a global framework that was better aligned with empirical observations on R\&D. The current paper extends this framework to R\&D cooperatives and is the first attempt to evaluate market collusion throughout all possible technological scenarios opened up by the global framework. On a methodological side, by considering a generalized dynamical system that also encompasses the single-firm model of Hinloopen et al. (2013), the paper for the first time analytically proves the existence of optimal solutions and indifference points in the global framework, thereby making a firm ground for further analyses and extensions.

One particular aspect of the analysis we have not explicitly addressed is the stability of cooperation. In the absence of the legal ban on price-fixing agreements (the counterfactual scenario this paper tries to assess), the firms in the model would have incentives to sign binding 
contracts as total discounted profits under full collusion are always at least as high as under partial (or no) collusion. However, even without such contracts, Yeung and Petrosyan (2004) show within the sphere of cooperative games with transferable payoffs (quite a reasonable assumption for firms engaged in R\&D cooperatives) that one can design a payoff distribution procedure ${ }^{26}$ that leads to a dynamically stable cooperation. More advanced considerations of cartel stability are not crucial to the arguments made in this paper and are therefore left for future work. It is reassuring, however, that none of the four technological outcomes appertaining to the bifurcation diagram is precluded by any given value of a discount rate per se. In practice usually not all firms in an industry are involved in the same $R \& D$ cooperative and unexpected changes in markets might make it necessary for firms to renegotiate their initial profit sharing agreements; they might also allow firms to mask their deviations. Entire R\&D cooperatives might fail, pushing firms into asymmetric development paths. A proper assessment of such possibilities necessarily calls for a global framework being extended to a situation in which potentially asymmetric firms compete in both dimensions - R\&D and price. We believe these are some fruitful areas for future research that have been opened up by the analysis in this paper.

\section{A Appendix}

\section{A.1 Proof of Proposition 1}

Second rescaling of the problem. Recall the dynamic optimization problem: to maximize

$$
\Pi=\int_{0}^{\infty}\left(\alpha(1-c)^{2} \chi(c)-k^{2}\right) \mathrm{e}^{-\rho t} \mathrm{~d} t
$$

subject to the dynamic restriction

$$
\dot{c}=(1-\phi(1+\beta) k) c .
$$

This problem is rewritten by introducing constants

$$
K=\frac{1}{\phi(1+\beta)} \quad \text { and } \quad \mu=\frac{\alpha \phi^{2}(1+\beta)^{2}}{4 \rho}
$$

and the variable $u$ through

$$
k=K u .
$$

It is then seen to be equivalent to the problem to maximize

$$
V=\frac{\Pi}{K^{2}}=\int_{0}^{\infty}\left(4 \rho \mu(1-c)^{2} \chi(c)-u^{2}\right) \mathrm{e}^{-\rho t} \mathrm{~d} t,
$$

\footnotetext{
${ }^{26}$ For instance, a sharing agreement according to which each firm receives a payoff equal to its noncooperative profits plus half of the profits in excess of noncooperative profits.
} 
subject to the dynamic restriction

$$
\dot{c}=(1-u) c
$$

and the control restriction

$$
u \geq 0
$$

The Pontryagin function of this problem is

$$
P=4 \rho \mu(1-c)^{2} \chi(c)-u^{2}+\lambda c(1-u)
$$

which is maximized at

$$
u=\max \left\{0,-\frac{c}{2} \lambda\right\}
$$

This yields the Hamilton function

$$
H=4 \rho \mu(1-c)^{2} \chi(c)+\lambda c+ \begin{cases}\frac{(\lambda c)^{2}}{4} & \text { if } \lambda \leq 0 \\ 0 & \text { if } \lambda>0\end{cases}
$$

If $\lambda \leq 0$, the associated state-costate equations read as

$$
\begin{aligned}
& \dot{c}=H_{\lambda}=\frac{\lambda c^{2}}{2}+c, \\
& \dot{\lambda}=\rho \lambda-H_{c}=\rho \lambda+8 \rho \mu(1-c) \chi(c)-\frac{\lambda^{2}}{2} c-\lambda,
\end{aligned}
$$

whereas if $\lambda>0$, they simplify to

$$
\dot{c}=c, \quad \dot{\lambda}=(\rho-1) \lambda+8 \rho \mu(1-c) \chi(c) .
$$

Using the relation (33) as a variable transformation whenever $\lambda \leq 0$, we can put the system into state-control form

$$
\begin{aligned}
\dot{c} & =F_{1}(c, u)=c(1-u), \\
\dot{u} & =F_{2}(c, u)=\rho(u-4 \mu c(1-c) \chi(c)) .
\end{aligned}
$$

For later use, we note that in $(c, u)$ variables, the Hamilton function takes the form

$$
H_{\text {control }}(c, u)=4 \rho \mu(1-c)^{2} \chi(c)+u^{2}-2 u \text {. }
$$

\section{A.1.1 Steady states}

To determine the steady states of the state-control system (37)-(38), we solve the equations $\dot{c}=0$, $\dot{u}=0$. It is immediate that this system has no solutions if $c>1$.

If $0 \leq c \leq 1$, the equation $\dot{c}=0$ is satisfied if $c=0$ or $u=1$. Substitution into $\dot{u}=0$ of the former yields the steady state $(c, u)=(0,0)$. Substitution of the latter leads to the quadratic 
equation

$$
c^{2}-c+\frac{1}{4 \mu}=0
$$

which can be written as

$$
\left(c-\frac{1}{2}\right)^{2}-D=0
$$

with

$$
D=\frac{1}{4}\left(1-\frac{1}{\mu}\right)
$$

Note that $D<\frac{1}{4}$, as all parameters are assumed to have positive values. For $D>0$, the quadratic equation has two real solutions

$$
c_{ \pm}=\frac{1}{2} \pm \sqrt{D}=\frac{1 \pm \sqrt{1-1 / \mu}}{2}
$$

both satisfying $0<c_{ \pm}<1$; for $D=0$, there is a single real solution $c=1 / 2$, while for $D<0$, there is no real solution.

Summarizing, if $0 \leq c \leq 1$, we have the steady states

$$
(c, u)=e_{0}=(0,0)
$$

and, for $D \geq 0$,

$$
(c, u)=e_{ \pm}=\left(c_{ \pm}, u_{ \pm}\right)=\left(\frac{1}{2} \pm \sqrt{D}, 1\right)
$$

\section{A.1.2 Stability}

To analyze stability, we have to determine the eigenvalues of

$$
D F=\left(\begin{array}{cc}
1-u & -c \\
4 \rho \mu(2 c-1) & \rho
\end{array}\right)
$$

at the steady states $e_{0}, e_{+}$and $e_{-}$. As

$$
D F\left(e_{0}\right)=\left(\begin{array}{cc}
1 & 0 \\
-4 \rho \mu & \rho
\end{array}\right)
$$

which has eigenvalues $\rho$ and 1 , the point $e_{0}$ is always an unstable node.

Denote the eigenvalues of the matrix

$$
D F\left(e_{ \pm}\right)=\left(\begin{array}{cc}
0 & -c_{ \pm} \\
\pm 8 \rho \mu \sqrt{D} & \rho
\end{array}\right)
$$


by $\lambda_{ \pm}^{i}, i=1,2$. They satisfy

$$
\lambda_{ \pm}^{1}+\lambda_{ \pm}^{2}=\operatorname{trace} D F\left(e_{ \pm}\right)=\rho
$$

and

$$
\lambda_{ \pm}^{1} \lambda_{ \pm}^{2}=\operatorname{det} D F\left(e_{ \pm}\right)= \pm 8 \rho \mu c_{ \pm} \sqrt{D} .
$$

We have seen before that $c_{ \pm}>0$ whenever it is real. If $D>0$, it follows that the eigenvalues $\lambda_{-}^{1}, \lambda_{-}^{2}$ have opposite sign, and $e_{-}$is a saddle, whereas $\lambda_{+}^{1}$ and $\lambda_{+}^{2}$ have the same sign and positive sum, implying that $e_{+}$is an unstable node.

Expressing these results in the original variables, we obtain the results announced in the proposition.

\section{A.1.3 Bifurcation analysis}

It remains to prove the occurrence of a saddle-node bifurcation. If $\mu=\mu_{b}=1$, then $D=0$ and the point $e_{b}=\left(c_{b}, u_{b}\right)=(1 / 2,1)$ is a steady state with eigenvalues 0 and $\rho$ respectively.

We use a result from Sotomayor (1973) (quoted as Theorem 3.4.1 in Guckenheimer and Holmes, 1986), which for planar dynamical systems states that if the family

$$
\dot{x}=F(x ; \mu)
$$

parametrised by $\mu$ satisfies the following three conditions

1. $D_{x} F\left(x_{0} ; \mu_{0}\right)$ has a simple eigenvalue 0 with right eigenvector $v$ and left eigenvector $w$;

2. $w D_{\mu} F\left(x_{0} ; \mu_{0}\right) \neq 0$;

3. $w\left[D_{x}^{2} F\left(x_{0} ; \mu_{0}\right)(v, v)\right] \neq 0$;

then it features a non-degenerate saddle-node bifurcation at $x=x_{0}$ for $\mu=\mu_{0}$.

As $D F\left(e_{b} ; \mu_{b}\right)=\left(\begin{array}{cc}0 & -1 / 2 \\ 0 & \rho\end{array}\right)$, it follows that $v=\left(\begin{array}{l}1 \\ 0\end{array}\right)$ and $w=\left(\begin{array}{ll}2 \rho & 1\end{array}\right)$ are respectively left and right eigenvectors associated to the eigenvalue 0 . Furthermore

$$
w D_{\mu} F\left(e_{b} ; \mu_{b}\right)=w\left(\begin{array}{c}
0 \\
-\rho
\end{array}\right)=-\rho \neq 0
$$

and, as $v=\left(\begin{array}{l}1 \\ 0\end{array}\right)$,

$$
w\left[D_{x}^{2} F\left(e_{b} ; \mu_{b}\right)(v, v)\right]=w \frac{\partial^{2}}{\partial c^{2}} F=w\left(\begin{array}{c}
0 \\
8 \rho
\end{array}\right)=8 \rho \neq 0 .
$$

We conclude that a nondegenerate saddle-node bifurcation occurs in the system at $\mu=1$. This completes the proof of Proposition 1. 


\section{A.2 Proof of Proposition 2}

As in the proof of Proposition 1, introduce the constants

$$
K=\frac{1}{\phi(1+\beta)} \quad \text { and } \quad \mu=\frac{\alpha \phi(1+\beta)}{4 \rho K}=\frac{\alpha \phi^{2}(1+\beta)^{2}}{4 \rho},
$$

as well as the rescaled control variable $u=k / K$. The state-control system then takes the form

$$
\dot{c}=c(1-u), \quad \dot{u}=\rho(u-4 \mu c(1-c) \chi(c)) .
$$

Recall also the notations

$$
e_{0}=(0,0), \quad e_{-}=\left(\frac{1-\sqrt{1-1 / \mu}}{2}, 1\right), \quad e_{+}=\left(\frac{1+\sqrt{1-1 / \mu}}{2}, 1\right)
$$

for the three steady states of the system, and introduce

$$
e_{1}=(1,0)
$$

To prove the proposition, the state-control space is partitioned into four subsets, $R_{1}, R_{2}, R_{3}$ and $E$. Of these, the sets $R_{3}$ and $E$ are independent of the values of the system parameters. They are given as

$$
R_{3}=\{(c, u): 0<c<1, u=0\}, \quad E=\{(c, u): c \geq 1, u=0\} .
$$

Let $U=\{(c, u): u>0\}$ be the upper half plane. Given the set $R_{1}$, the set $R_{2}$ is equal to

$$
R_{2}=U \backslash R_{1}
$$

It remains to specify $R_{1}$, which is the first step in the proof. Then it is shown that no trajectory in either $R_{2}$ or $R_{3}$ can be optimal. The next step is to demonstrate that of the trajectories in $R_{1}$, only those can be optimal which converge either to a steady state in $R_{1}$, necessarily a saddle, or which end up in the "exit trajectory" $E$. Then it has to be shown that the trajectories that are not excluded up to this point, the candidate trajectories, "cover" the state space; that is, for every initial state $c_{0}$, there is at least one candidate trajectory passing through the line $c=c_{0}$. Using parts of the remaining candidate trajectories, we construct a viscosity solution of the HamiltonJacobi equation, which is then necessarily the value function. This shows the optimality of the remaining trajectories.

\section{A.2.1 Definition of $R_{1}$}

Set

$$
u_{0}=\max \{1, \mu\}
$$


and consider the trajectory $\gamma(t)=(c(t), u(t))$ of the system (43) that passes at $t=0$ through the point $\left(1, u_{0}\right)$.

If $\mu \leq 1$, then $u_{0}=1$ and $R_{1}$ is specified as

$$
R_{1}=\{(c, u): 0 \leq c \leq 1,0<u \leq 1\}
$$

If the other possibility $\mu>1$ obtains, then $u_{0}=\mu>1$ and $\dot{c}(0)<0$. In this situation, let $\tau$ be the least upper bound of those negative values of $t$ that satisfy $c(t) \leq 1$; that is, let

$$
\tau=\sup \{t<0: c(t) \leq 1\}
$$

We claim that $\tau$ is finite. Arguing by contradiction, assume that $\tau=-\infty$. Then for all $t<0$ we have $c(t)>1$, and equation (43) implies that for all $t<0$

$$
u(t)=u_{0} \mathrm{e}^{\rho t} .
$$

In particular, there is a $t_{1}<0$ such that

$$
u(t)<u_{0} \mathrm{e}^{\rho t_{1}}=: K_{1}<1
$$

for all $t<t_{1}$. But for those values of $t$, it follows that

$$
\dot{c}=(1-u) c>\left(1-K_{1}\right) c=: K_{2} c
$$

where $K_{2}>0$. Gronwall's lemma implies then that

$$
c(t)<\mathrm{e}^{K_{2}\left(t-t_{1}\right)} c\left(t_{1}\right)
$$

if $t<t_{1}$. But for $t$ sufficiently small, this is smaller than 1 , contradicting the hypothesis that $\tau=-\infty$. Hence $\tau$ is finite.

Introduce $u_{\tau}$ by the equation $\gamma(\tau)=\left(1, u_{\tau}\right)$. The set $R_{1}$ is defined as follows: it is the open region bounded by the concatenation of the curve $\gamma$ taken between $t=0$ and $t=\tau$, connecting $\left(1, u_{0}\right)$ and $\left(1, u_{\tau}\right)$, the vertical line segment connecting $\left(1, u_{\tau}\right)$ to $e_{1}$, the horizontal segment connecting $e_{1}$ to $e_{0}$, the vertical segment connecting $e_{0}$ to $\left(0, u_{0}\right)$, and the horizontal segment connecting $\left(0, u_{0}\right)$ to $\left(1, u_{0}\right)$. See Figure 10 for the possible shapes of $R_{1}$.

\section{A.2.2 Trajectories in $R_{2}$ cannot be optimal}

In the second step of the proof, the transversality condition is used to show that any trajectory that passes through points in $R_{2}$ cannot be optimal.

Beginning with $R_{2}$, we note that the subset

$$
R_{2}^{(1)}=\{(c, u): 0 \leq c \leq 1\} \cap R_{2}
$$




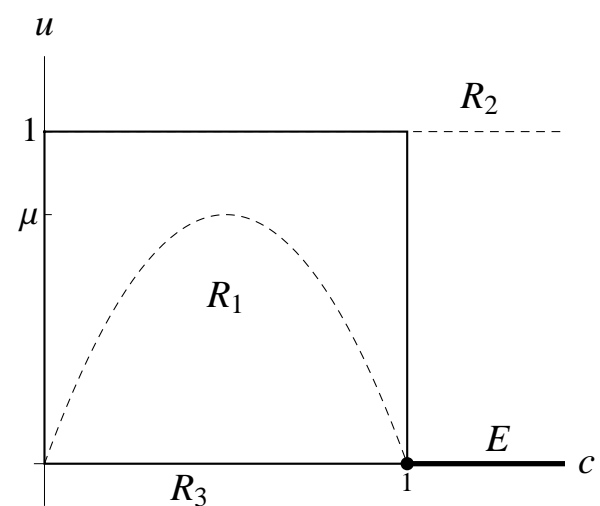

(a) $\mu \leq 1$

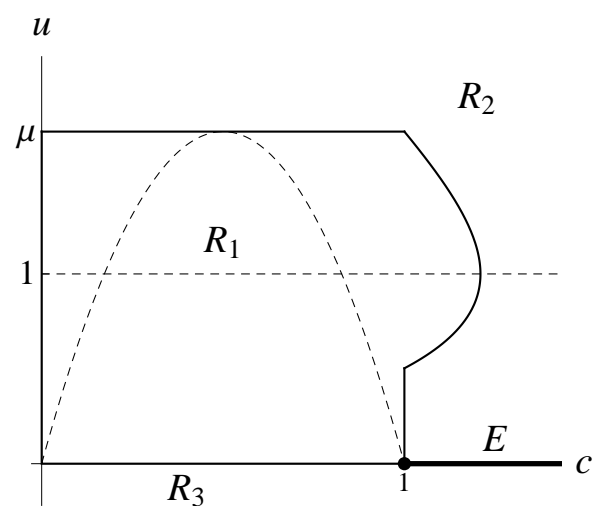

(b) $\mu>1$

Figure 10: Definition of the set $R_{1}$. Solid curves denote the boundary of the set, dashed curves the isoclines of the system (37)-(38).

of $R_{2}$ is a forward trapping region: once a trajectory of (43) is inside $R_{2}^{(1)}$, it remains inside for all subsequent times. This fact is established by demonstrating that the vector field defined by (43) is inward pointing on the boundary of $R_{2}^{(1)}$. For, if $u=u_{0}=\max \{1, \mu\}$ and $0 \leq c \leq 1$, then

$$
\dot{u} \geq \rho(\mu-4 \mu c(1-c))=0 .
$$

If $c=0$, then $\dot{c}=0$, and if finally $c=1$ and $u \geq u_{0} \geq 1$, then

$$
\dot{c} \leq c(1-1)=0 \text {. }
$$

Actually, we can make the sharper statement that if $u>u_{0}$, then

$$
\dot{u}>0 \text {. }
$$

To show that no trajectory that enters $R_{2}^{(1)}$ can be maximizing, pick an arbitrary trajectory $\gamma$ such that $\gamma\left(t_{0}\right) \in R_{2}^{(1)}$ at a given time $t_{0}$. By the Poincaré-Bendixon theorem, $\gamma(t)$ is either unbounded, or its $\omega$-limit set is a steady state, or a limit cycle. The latter possibility is excluded, as the state-costate system, which is in one-to-one relation with the state-control system, has constant positive divergence everywhere (see Wagener, 2003). There are no steady states in $R_{2}^{(1)}$. Hence there is a sequence $t_{0}, t_{1}, \ldots$ such that $\left\|\gamma\left(t_{i}\right)\right\| \rightarrow \infty$. In particular, there is $\bar{t}>t_{0}$ such that $u(\bar{t})>2 u_{0}$. But then $u(t)$ is monotonely increasing towards infinity as $t>\bar{t}$, as a consequence of (44).

Consequently, if $t \geq \bar{t}$, then

$$
\dot{c} \leq\left(1-2 u_{0}\right) c \leq-c .
$$


By Gronwall's lemma it follows that

$$
c(t) \leq c(\bar{t}) \mathrm{e}^{-(t-\bar{t})}
$$

Likewise, if $t \geq \bar{t}$, then $u(t)>2 u_{0}$ and

$$
\dot{u} \geq \rho(u-\mu) .
$$

Gronwall's lemma implies then that

$$
u(t) \geq \mu+\left(2 u_{0}-\mu\right) \mathrm{e}^{\rho(t-\bar{t})} .
$$

If the trajectory $\gamma(t)=(c(t), u(t))$ is optimal, then by the Hamilton-Jacobi equation (see e.g. Wagener, 2003), the total profit $\Pi$ takes the value

$$
\Pi(c(0))=\frac{1}{\rho} H(c(0), \lambda(0))=\frac{1}{\rho} H_{\text {control }}(c(0), u(0)) .
$$

Michel's transversality condition (Michel, 1982) states that along a maximizing trajectory the relation

$$
\lim _{t \rightarrow \infty} \Pi(c(t)) \mathrm{e}^{-\rho t}=0
$$

holds. Combining (47) and (39) yields

$$
\Pi(c(t)) \mathrm{e}^{-\rho t} \geq\left(4 \rho \mu(1-c(t))^{2} \chi(c(t))+u(t)(u(t)-2)\right) \mathrm{e}^{-\rho t}
$$

Using that the first term between brackets is always nonnegative, and taking into account (46) yields that

$$
\Pi \mathrm{e}^{-\rho t} \geq\left(2 u_{0}-\mu\right) \mathrm{e}^{\rho(t-\bar{t})}\left(\mu-2+\left(2 u_{0}-\mu\right) \mathrm{e}^{\rho(t-\bar{t})}\right) \mathrm{e}^{-\rho t} .
$$

As $2 u_{0}-\mu \geq \mu>0$, it follows that the right hand side of this inequality tends to infinity as $t \rightarrow \infty$. But then

$$
\lim _{t \rightarrow \infty} \Pi(c(t)) \mathrm{e}^{-\rho t}=\infty,
$$

and $\gamma$ cannot be a maximizing trajectory.

It remains to show that no trajectory passing through

$$
R_{2}^{(2)}=R_{2} \backslash R_{2}^{(1)}
$$

the complement of $R_{2}^{(1)}$ in $R_{2}$, can be optimal. Consider therefore a trajectory $\gamma$ such that $\gamma\left(t_{0}\right) \in$ $R_{2}^{(2)}$ for some $t_{0}$. As in the definition of the region $R_{1}$, using Gronwall's lemma it can be shown that there is some $t_{1}>t_{0}$ such that $u\left(t_{1}\right)>1$, and some $t_{2}>t_{1}$ such that $u\left(t_{2}\right)>1$ 
and $c\left(t_{2}\right)=1$. But then $\gamma$ enters the trapping region $R_{2}^{(1)}$, and we have already seen that such trajectories cannot be optimal.

\section{A.2.3 Trajectories intersecting $R_{3}$ cannot be optimal}

If a trajectory intersects $R_{3}$, the state-control representation breaks down, and we have to switch to the state-costate representation.

Pick an arbitrary state-costate trajectory $\gamma(t)=(c(t), \lambda(t))$, with associated control $u(t)=$ $\max \left\{0,-\frac{1}{2} c(t) \lambda(t)\right\}$ such that $\left(c\left(t_{0}\right), u\left(t_{0}\right)\right) \in R_{3}$ for some $t_{0} \geq 0$ and $(c(t), u(t)) \in R_{1}$ for all $t<t_{0}$ that are sufficienty close to $t_{0}$. The costate $\lambda$ then satisfies $\lambda\left(t_{0}\right)=0$ and $\dot{\lambda}\left(t_{0}\right)>0$. Note that the region

$$
\tilde{R}_{3}=\{(c, \lambda): \lambda>0\}
$$

is a trapping region for the state-costate flow, as $\dot{\lambda} \geq 0$ whenever $\lambda=0$.

Using Gronwall's lemma, we show first that

$$
c(t) \geq c\left(t_{0}\right) \mathrm{e}^{\left(t-t_{0}\right)},
$$

for $t>t_{0}$, since $\dot{c}=c \geq c$ in $\tilde{R}_{3}$ (equation (36)). It follows that there is $t_{1}>t_{0}$ such that $c(t)>1$ for all $t>t_{1}$.

Let $h(t)=H(c(t), \lambda(t))$. Note that for all $t>t_{1}$ we have $c(t)>1$ and $\lambda(t)>0$, and consequently $h(t)=\lambda(t) c(t)>0$. The state-costate equations reduce to

$$
\dot{c}=c, \quad \dot{\lambda}=(\rho-1) \lambda .
$$

Compute:

$$
\dot{h}=\dot{\lambda} c+\lambda \dot{c}=\rho \lambda c=\rho h .
$$

Hence

$$
h(t)=h\left(t_{1}\right) \mathrm{e}^{\rho\left(t-t_{1}\right)}
$$

for all $t>t_{1}$. But then

$$
\lim _{t \rightarrow \infty} h(t) \mathrm{e}^{-\rho t}=h\left(t_{1}\right) \mathrm{e}^{-\rho t_{1}}>0 .
$$

If $\gamma$ is optimal, Michel's transversality condition implies that

$$
\lim _{t \rightarrow \infty} \Pi(c(t)) \mathrm{e}^{-\rho t}=\lim _{t \rightarrow \infty} \frac{1}{\rho} H(c(t), \lambda(t)) \mathrm{e}^{-\rho t}=\lim _{t \rightarrow \infty} \frac{h(t)}{\rho} \mathrm{e}^{-\rho t}=0 .
$$

As this is a contradiction, the trajectory $\gamma$ cannot be optimal. 


\section{A.2.4 Trajectories in $R_{1}$ with wrong limit behavior cannot be optimal}

As the set $R_{1}$ is bounded, by the Poincaré-Bendixon theorem trajectories in $R_{1}$ can either converge to a steady state, or leave $R_{1}$ (cf. the argument in Section A.2.2). Those entering either $R_{2}$ or $R_{3}$ have already been shown to be suboptimal. The remaining possibility is to leave $R_{1}$ through the point $e_{1}$ and enter the line segment $E$; these trajectories remain candidates for optimality.

Trajectories remaining in $R_{1}$ have to converge to a steady state. From proposition 1 we learn that $e_{0}$ and $e_{+}$are unstable nodes, to which no trajectory can converge as $t \rightarrow \infty$. The only remaining candidate is then the saddle $e_{-}$, if $\mu<1$, or the bifurcating point $e_{b}$ if $\mu=1$.

This completes the proof of Proposition 2.

\section{A.3 Proof of proposition 3}

\section{A.3.1 Construction of policy functions}

The first step in the proof is to construct those (parts of) trajectories of the system (43) that will turn out to optimize the profit functional. In particular, we shall construct a, possibly multivalued, policy function $u_{f}$ such that the following holds. If $\left(c_{0}, u_{0}\right)$ is such that $u_{0}=u_{f}\left(c_{0}\right)$, then the trajectory $(c(t), u(t))$ of (43) starting at this point satisfies, for all $t \geq 0$, that $\dot{c}(t) \neq 0$ and $u(t)=u_{f}(c(t))$.

Again we have to distinguish between the situations $\mu<1$ and $\mu \geq 1$.

First situation: $\mu<1$. Here the only steady state of (43) is the origin $e_{0}$, which is an unstable node. Therefore, the only candidate optimizer is the trajectory passing through the point $e_{1}$. Note that a corollary of the analysis performed above is that the set $R_{1}$ is a backward trapping region: if a trajectory is in $R_{1}$ for some time, it is in $R_{1}$ for all previous times. Necessarily it converges to the origin as $t \rightarrow-\infty$.

Let $\gamma(t)=\left(c_{\gamma}(t), u_{\gamma}(t)\right)$ be the trajectory such that $\gamma(0)=e_{1}$. As $\gamma(t) \in R_{1}$ for all $t<0$, it follows that $\dot{c}_{\gamma}>0$ for all $t<0$ (recall that $R_{1}$ is open). As $u(t)=0$ for all $t \geq 0$, it follows that $\dot{c}_{\gamma}>0$ for all $t$, and that the map $c_{\gamma}: \mathbb{R} \rightarrow(0, \infty)$ is invertible, with inverse $t=t_{\gamma}(c)$. Define $u_{f}:(0, \infty) \rightarrow \mathbb{R}$ by

$$
u_{f}(c)=u_{\gamma}\left(t_{\gamma}(c)\right)
$$

Then the image of the curve $\gamma: \mathbb{R} \rightarrow \mathbb{R}^{2}$ is equal to the graph of the function $u_{f}:(0, \infty) \rightarrow \mathbb{R}$, as

$$
u_{\gamma}(t)=u_{f}\left(c_{\gamma}(t)\right)
$$

for all $t$.

Second situation: $\mu \geq 1$. In this case, though $R_{1}$ is still a backward trapping region, there are at least two steady states in $R_{1}$ : apart from the origin $e_{0}$, we also have $e_{-}$and $e_{+}$. As seen before, if $D>0$, the first is a saddle and the second a repeller; if $D=0$, these two points coincide in $e_{b}$. 
Denote by $\delta_{1}$ the part of the parabola $u=4 \mu c(1-c)$ connecting $e_{0}$ to $e_{-}$, by $\delta_{2}$ the segment of the line $u=1$ connecting $e_{-}$to $e_{+}$, by $\delta_{3}$ that part of the same parabola which connects $e_{+}$ to $e_{1}$, and by $\delta_{4}$ the segment of the line $u=0$ connecting $e_{1}$ to $e_{0}$. All curves $\delta_{i}$ are taken without their endpoint. Let finally $S_{1} \subset R_{1}$ be the open subregion of $R_{1}$ that is bounded by the curves $\delta_{i}$, $i=1, \ldots, 4$. See Figure 11 .

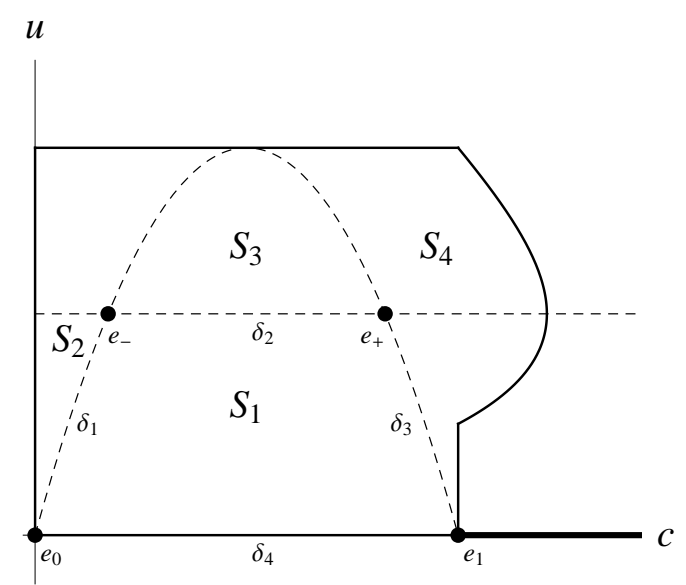

Figure 11: Subdivision of region $R_{1}$. The vertices $e_{0}, e_{1}, e_{-}$and $e_{+}$, the edges $\delta_{i}, i=1, \ldots, 4$, and the faces $S_{i}, i=1, \ldots, 4$ are defined in the text.

Let $\gamma(t)=(c(t), u(t))$ be the trajectory of (43) satisfying $\gamma(0)=e_{1}$. As the open set $S_{1}$ is bounded, the trajectory $\gamma$ either converges to a steady state on the boundary of $S_{1}$, or it enters $S_{1}$ for the last time by crossing one of the curves $\delta_{i}$. We analyze the possibilities one by one.

The trajectory remains in $S_{1}$ for all $t<0$ and tends to $e_{0}$. If $\gamma(t) \in S_{1}$ for all $t<0$ and $\gamma(t) \rightarrow e_{0}$ as $t \rightarrow-\infty$, then the results of the situation $D<0$ carry over unmodified, and we obtain a policy function $u_{f}:(0, \infty) \rightarrow \mathbb{R}$.

The trajectory remains in $S_{1}$ for all $t<0$ and tends to $e_{-}$. If $\gamma(t) \in S_{1}$ for all $t<0$ and $\gamma(t) \rightarrow e_{-}$as $t \rightarrow-\infty$, then $\gamma$ is part of the unstable manifold of $e_{-}$. Reasoning as in the situation $D<0$, we obtain a policy function

$$
u_{f}^{(1)}:\left(c_{-}, \infty\right) \rightarrow \mathbb{R}
$$

with

$$
\lim _{c \downarrow c_{-}} u_{f}^{(1)}(c)=u_{-}=1 .
$$

However, this function is not defined for all $c>0$. To construct a policy function for $0<c<c_{-}$, we take a trajectory $\gamma^{s}$ on the left half of the stable manifold of the saddle $e_{-}$.

We claim that this part of the stable manifold is contained in its entirety in the region $S_{2}$ that is bounded by $\delta_{1}$, the segment of $u=1$ connecting $e_{-}$to $(0,1)$, and the segment of the line $c=0$ connecting $(0,1)$ to $e_{0}$. It is straightforward to show that $S_{2}$ is a backward trapping 
region; consequently, every trajectory in $S_{2}$ converges to the unstable node $e_{0}$ as $t \rightarrow-\infty$.

The stable manifold of $e_{-}$is tangent to the stable eigenspace of

$$
D F\left(e_{-}\right)=\left(\begin{array}{cc}
0 & -c_{-} \\
8 \rho \mu \sqrt{D} & \rho
\end{array}\right),
$$

cf. equation (42), at $e_{-}$. Note that the vector $v=(0,1)$ cannot be an eigenvector of this matrix, as $c_{-} \neq 0$. Therefore any eigenvector $v=\left(v_{1}, v_{2}\right)$ satisfies $v_{1} \neq 0$; it may therefore be assumed that $v_{1}=1$.

Let $v^{s}=\left(1, v_{2}^{s}\right)$ be the stable eigenvector, with eigenvalue $\lambda^{s}<0$. The eigenvalue equation

$$
D F\left(e_{-}\right) v^{s}=\lambda^{s} v^{s}
$$

then yields

$$
v_{2}^{s}=-\frac{\lambda^{s}}{c_{-}}>0
$$

Locally around the saddle, the stable manifold coincides with the graph of a function $w^{s}$, defined on a neighborhood of $c_{-}$, which is of the form

$$
w^{s}(c)=c_{-}+v_{2}^{s}\left(c-c_{-}\right)+O\left(\left(c-c_{-}\right)^{2}\right)
$$

In particular, if $c_{0}<c_{-}$is sufficiently close to $c_{-}$, then

$$
\frac{\mathrm{d} w^{s}}{\mathrm{~d} c}(c)>0
$$

for all $c \in\left[c_{0}, c_{-}\right]$. The trajectory $\gamma(t)$ of (43) such that $\gamma(0)=\left(c_{0}, w^{s}\left(c_{0}\right)\right)$ consequently satisfies $c_{0} \leq c(t)<c_{-}$, as well as $\dot{c}(t)>0$ and $\dot{u}(t)>0$ for all $t \geq 0$. We infer that necessarily

$$
4 \mu c(t)(1-c(t))<u(t)<1
$$

for all $t \geq 0$, and hence $(c(t), u(t)) \in S_{2}$ for all $t \geq 0$. But as $S_{2}$ is a backward trapping region, the trajectory $\gamma$ is contained in $S_{2}$ for all $t$, hence satisfying

$$
\gamma(t) \rightarrow e_{0} \quad \text { as } \quad t \rightarrow-\infty, \quad \text { and } \quad \gamma(t) \rightarrow e_{-} \quad \text { as } \quad t \rightarrow \infty .
$$

As in $S_{2}$, we have $\dot{c}>0$ everywhere, and we construct as above a policy function

$$
u_{f}^{(2)}:\left(0, c_{-}\right) \rightarrow \mathbb{R}, \quad \text { with } \quad \lim _{c \uparrow c_{-}} u_{f}^{(2)}(c)=u_{-}=1 .
$$


It follows that the function

$$
u_{f}(c)= \begin{cases}u_{f}^{(1)}(c) & \text { if } c>c_{-}, \\ u_{-} & \text {if } c=c_{-}, \\ u_{f}^{(2)}(c) & \text { if } 0<c<c_{-},\end{cases}
$$

is a continuous policy function that is defined for all $c>0$.

The trajectory remains in $S_{1}$ for all $t<0$ and tends to $e_{+}$. As before, we can construct a policy function

$$
u_{f}^{(1)}:\left(c_{+}, \infty\right) \rightarrow \mathbb{R}, \quad \text { with } \quad \lim _{c \downarrow c_{+}} u_{f}^{(1)}(c)=u_{+}=1 .
$$

The remaining part of the policy function has to be furnished by the stable manifold of $e_{-}$. As above, the left half of this stable manifold furnishes a policy function

$$
u_{f}^{(2)}:\left(0, c_{-}\right) \rightarrow \mathbb{R}, \quad \text { with } \quad \lim _{c \uparrow c_{-}} u_{f}^{(2)}(c)=u_{-}=1 .
$$

We turn to the right half of the stable manifold. For values of $c_{0}$ larger than but close to $c_{-}$, the point $\left(c_{0}, u_{0}\right)=\left(c_{0}, w^{s}\left(c_{0}\right)\right)$ on the stable manifold is contained in the bounded open region $S_{3}$ that is bounded by the line $u=1$ and the parabola $u=4 \mu c(1-c)$. In this region $\dot{c}<0$ and $\dot{k}<0$. Fix $\left(c_{0}, u_{0}\right)$ and consider the trajectory $\gamma$ of (43) such that $\gamma(0)=\left(c_{0}, u_{0}\right)$. This trajectory enters $S_{3}$ through the part of the parabola connecting its vertex $(1 / 2, \mu)$ with the point $e_{+}$. It enters from the region $S_{4}$ that is bounded by that same part of the parabola, the line $u=u_{+}$and the boundary of $R_{1}$. In that region, $\dot{c}<0$, but $\dot{k}>0$. It follows that the trajectory has to enter $S_{4}$ through the line segment of $c=c_{+}$connecting $e_{+}$and $\left(c_{+}, \mu\right)$, or through one of the endpoints.

If $\gamma(t) \rightarrow e_{+}$as $t \rightarrow-\infty$, then its graph defines a policy function

$$
u_{f}^{(3)}:\left(c_{-}, c_{+}\right) \rightarrow \mathbb{R} \quad \text { with } \quad \lim _{c \downarrow c_{-}} u_{f}^{(3)}(c)=u_{-}=1, \quad \lim _{c \uparrow c_{+}} u_{f}^{(3)}(c)=u_{+}=1 .
$$

A continuous policy function is then given by

$$
u_{f}(c)= \begin{cases}u_{f}^{(1)}(c) & \text { if } c>c_{+}, \\ u_{+} & \text {if } c=c_{+}, \\ u_{f}^{(2)}(c) & \text { if } 0<c<c_{-}, \\ u_{-} & \text {if } c=c_{-}, \\ u_{f}^{(3)}(c) & \text { if } c_{-}<c<c_{+} .\end{cases}
$$

Otherwise, there is a time $t_{1}<0$, such that $c\left(t_{1}\right)=c_{+}$and $u\left(t_{1}\right)>u_{+}$. As in this case $\gamma(t)$ does not tend to a steady state in the boundary of $S_{4}$, it has to enter $S_{4}$ for some $t_{2}<t_{1}$; the only 
possibility for this is through the line $u=1$. We therefore have

$$
\gamma\left(t_{2}\right)=\left(c_{M}, 1\right)
$$

In this situation, the graph $\gamma\left(\left[t_{2}, \infty\right)\right)$ defines a policy function

$$
u_{f}^{(3)}:\left(c_{-}, c_{M}\right) \rightarrow \mathbb{R} \quad \text { with } \quad \lim _{c \downarrow c_{-}} u_{f}^{(3)}(c)=u_{-}=1, \quad \lim _{c \uparrow c_{M}} u_{f}^{(3)}(c)=1 .
$$

On the interval $\left(c_{+}, c_{M}\right)$, there are now two policy functions defined. Recall that the total profit at an initial state $c$ of an $\mathrm{R} \& \mathrm{D}$ policy for which $u=u_{f}(c)$ is given by

$$
\Pi(c)=\frac{1}{\rho} H_{\text {control }}(c, u)=\frac{1}{\rho}\left(4 \rho \mu(1-c)^{2} \chi(c)+u^{2}-2 u\right) .
$$

For fixed values of $c$, the function $H_{\text {control }}(c, u)$ is minimal at $u=1$. Hence the policy $u_{f}^{(3)}$ is superior to $u_{f}^{(1)}$ at $c=c_{+}$, but it is inferior to it at $c=c_{M}$. As both functions are continuous, there is a value $c=\hat{c}$ such that both policies generate the same total profit. This is an indifference point, as the manager is indifferent between two policies at this state. A policy function, which is at one point two-valued, is then given by

$$
u_{f}(c)= \begin{cases}u_{f}^{(1)}(c) & \text { if } c>\hat{c}, \\ u_{f}^{(1)}(\hat{c}) \text { or } u_{f}^{(3)}(\hat{c}) & \text { if } c=\hat{c}, \\ u_{f}^{(2)}(c) & \text { if } 0<c<c_{-}, \\ u_{-} & \text {if } c=c_{-}, \\ u_{f}^{(3)}(c) & \text { if } c_{-}<c<\hat{c} .\end{cases}
$$

Note that the induced total profit $\Pi(c)=H_{\text {control }}\left(c, u_{f}(c)\right) / \rho$ is Lipschitz continuous.

The trajectory enters $S_{1}$ for the last time through $\delta_{1}$. The next situation to be investigated is that the trajectory $\gamma$ satisfying $\gamma(0)=e_{1}$ enters $S_{1}$ through $\delta_{1}$ at some time $t_{1}<0$, and remains in $S_{1}$ for all $t_{1}<t<0$. But then it has to be in the backward trapping region $S_{2}$ for all $t<t_{1}$, and it converges to $e_{0}$ as $t \rightarrow-\infty$. As $\dot{c}>0$ in both $S_{1}$ and $S_{2}$, we can construct a differentiable policy function exactly as in the situation that the trajectory remains in $S_{1}$ for $t<0$ and converges to $e_{0}$.

The trajectory enters $S_{1}$ for the last time through $\delta_{2}$. Finally consider the situation that the trajectory $\gamma$ that passes through $e_{1}$ at $t=0$ enters $S_{1}$ through $\delta_{2}$ for some $t_{1}<0$, and remains in $S_{1}$ for all $t_{1}<t<0$. Introduce $c_{m}$ by setting $\gamma\left(t_{1}\right)=\left(c_{m}, 1\right)$. As $\dot{c}(t)>0$ for $t_{1}<t<0$ as well as for $t \geq 0$, we can construct a continuous policy function

$$
u_{f}^{(1)}:\left[c_{m}, \infty\right) \rightarrow \mathbb{R}, \quad u_{f}^{(1)}\left(c_{m}\right)=1
$$


in the usual manner. The left branch of the stable manifold of the saddle $e_{-}$furnishes a continuous policy function

$$
u_{f}^{(2)}:\left(0, c_{-}\right) \rightarrow \mathbb{R}, \quad \text { with } \quad \lim _{c \uparrow c_{-}} u_{f}^{(2)}(c)=u_{-}=1,
$$

and the right branch of that manifold furnishes a continuous policy function

$$
u_{f}^{(3)}:\left(c_{-}, c_{M}\right) \rightarrow \mathbb{R}, \quad \text { with } \quad \lim _{c \downarrow c_{-}} u_{f}^{(3)}(c)=u_{-}=1, \quad u_{f}^{(3)}\left(c_{M}\right)=1,
$$

where $c_{+} \leq c_{M}$. Invoking the same arguments as above, we show that $u_{f}^{(3)}$ is superior to $u_{f}^{(1)}$ at $c=c_{m}$ and inferior to it at $c=c_{M}$. By the intermediate value theorem, there is an indifference point $\hat{c}$ such that $c_{m}<\hat{c}<c_{M}$, and such that the manager is indifferent between the two policies at $c=\hat{c}$. A policy function defined on all points of state space is then

$$
u_{f}(c)= \begin{cases}u_{f}^{(1)}(c) & \text { if } c>\hat{c} \\ u_{f}^{(1)}(\hat{c}) \text { or } u_{f}^{(3)}(\hat{c}) & \text { if } c=\hat{c} \\ u_{f}^{(2)}(c) & \text { if } 0<c<c_{-}, \\ u_{-} & \text {if } c=c_{-}, \\ u_{f}^{(3)}(c) & \text { if } c_{-}<c<\hat{c} .\end{cases}
$$

Summary. For all parameters, we have constructed a policy function

$$
u_{f}:(0, \infty) \rightarrow \mathbb{R}
$$

which is single-valued, except at most at one point $\hat{c}$, the indifference point. Moreover, the values of the two trajectories originating at an indifference point are the same.

\section{A.3.2 Policy functions generate viscosity solutions of the Hamilton-Jacobi equation}

Using relation (47), we have that

$$
V(c)=\frac{1}{\rho} H_{\text {control }}\left(c, u_{f}(c)\right)
$$

is well-defined at $c=\hat{c}$, continuous and continuously differentiable at all points $c>0$ except $\hat{c}$. Moreover, the value of the total profit (32) along a trajectory $\gamma$ of the state-control system (37) such that $\gamma(0)=\left(c, u_{f}(c)\right)$ is equal to $V(c)$.

We now go back to the state-costate representation (34)-(35), and introduce the feedback costate function

$$
\lambda_{f}(c)=-\frac{2}{c} u_{f}(c)
$$

Note that then

$$
V(c)=\frac{1}{\rho} H\left(c, \lambda_{f}(c)\right)
$$


By construction, if $\gamma(t)=(c(t), \lambda(t))$ is a solution of the state-costate system such that $\lambda(0)=$ $\lambda_{f}(c(0))$, then

$$
\lambda(t)=\lambda_{f}(c(t)) \text { for all } t
$$

If $t>0$, then $c(t) \neq \hat{c}$ and $\lambda_{f}$ is differentiable at $c(t)$; by the chain rule

$$
\dot{\lambda}=\lambda_{f}^{\prime}(c) \dot{c}
$$

We claim that $\lambda_{f}(c)=V^{\prime}(c)$ for all $c \neq \hat{c}$. For, differentiating (49) with respect to $c$ yields

$$
V^{\prime}(c)=\frac{1}{\rho}\left(H_{c}+H_{\lambda} \lambda_{f}^{\prime}(c)\right) \text {. }
$$

Evaluating this equation at $c=c(t)$, using first (50) and then (34) and (35) gives

$$
\begin{aligned}
V^{\prime}(c(t)) & =\frac{1}{\rho}\left(H_{c}+H_{\lambda} \frac{\dot{\lambda}}{\dot{c}}\right) \\
& =\frac{1}{\rho}\left(H_{c}+H_{\lambda} \frac{\rho \lambda-H_{c}}{H_{\lambda}}\right) \\
& =\lambda(t)=\lambda_{f}(c(t)) ;
\end{aligned}
$$

this proves the claim.

It follows that the function $V$ defined by (49) is a regular solution of the Hamilton-Jacobi equation

$$
\rho V(c)=H\left(c, V^{\prime}(c)\right)
$$

for all $c \neq \hat{c}$.

Viscosity solutions. We quote the definition of viscosity sub- and supersolutions from Fleming and Soner (2006, section II.11, p. 106).

\section{Definition}

$1^{\circ}$ A function $W$ is a viscosity subsolution of (51) at $\bar{c}$, if for every continuously differentiable function $w$ such that the difference $W-w$ takes a local maximum at $\bar{c}$, we have

$$
\rho V(\bar{c})-H\left(\bar{c}, w^{\prime}(\bar{c})\right) \leq 0
$$

$2^{\circ}$ A function $W$ is a viscosity supersolution of (51) at $\bar{c}$, if for every continuously differentiable function $w$ such that the difference $W-w$ takes a local minimum at $\bar{c}$, we have

$$
\rho V(\bar{c})-H\left(\bar{c}, w^{\prime}(\bar{c})\right) \geq 0
$$

$3^{\circ}$ A function $W$ is a viscosity solution of (51), if it is both a viscosity subsolution and a viscosity 


\section{supersolution.}

As $V$ is continuously differentiable in the neighborhood of every point $\bar{c} \neq \hat{c}$, taking $w=V$ in these definitions shows that $V$ is a viscosity solution of the Hamilton-Jacobi equation (51) at $\bar{c}$ if and only if it is a regular solution at $\bar{c}$.

It remains to show that $V$ is a viscosity solution at an indifference point $\hat{c}$. Note that the left and right limits of $V^{\prime}(c)$ exist at $\hat{c}$; we write

$$
\hat{\lambda}^{-}=\lim _{c \uparrow \hat{c}} V^{\prime}(c), \quad \hat{\lambda}^{+}=\lim _{c \downarrow \hat{c}} V^{\prime}(c) .
$$

From the analysis done above, we infer that

$$
\hat{\lambda}^{-}<\hat{\lambda}^{+}
$$

Let $v$ be a continuously differentiable function such that $V-v$ takes a local minimum at $c=\hat{c}$. Then necessarily

$$
\lim _{c \uparrow \hat{c}} V^{\prime}(c)-v^{\prime}(c) \leq 0, \quad \lim _{c \downarrow \hat{c}} V^{\prime}(c)-v^{\prime}(c) \geq 0,
$$

implying that

$$
\hat{\lambda}^{-} \leq v^{\prime}(c) \leq \hat{\lambda}^{+} .
$$

As $\hat{c}$ is an indifference point, we have that

$$
H\left(\hat{c}, \hat{\lambda}^{-}\right)=H\left(\hat{c}, \hat{\lambda}^{+}\right)=\rho V(\hat{c}) .
$$

Moreover, the Hamilton function $H(c, \lambda)$ is convex in $\lambda$. Together with (54) this implies that

$$
\rho V(\hat{c})-H\left(\hat{c}, v^{\prime}(\hat{c})\right) \geq 0 .
$$

Hence $V$ is a viscosity supersolution.

Consider now the situation that $v$ is a continuously differentiable function such that $V-v$ takes a local maximum at $\hat{c}$. Then

$$
\lim _{c \uparrow \hat{c}} V^{\prime}(c)-v^{\prime}(c) \geq 0, \quad \lim _{c \downarrow \hat{c}} V^{\prime}(c)-v^{\prime}(c) \leq 0,
$$

which implies that

$$
v^{\prime}(\hat{c}) \leq \hat{\lambda}_{-}<\hat{\lambda}_{+} \leq v^{\prime}(\hat{c}),
$$

which is a contradiction. There is no differentiable function such that $V-v$ takes a local minimum; but then for all such functions, the inequality (52) holds at $\hat{c}$, and $V$ is a viscosity subsolution.

As we know (cf. Fleming and Soner, 2006) that the unique viscosity solution of the HamiltonJacobi equation is the value function of the problem, it follows that the trajectories defined by the policy function are optimal. This concludes the proof. 


\section{A.4 Proof of Proposition 4}

This is an immediate consequence of the scaling (31). For assume that there is a bifurcation at $(\mu, \rho)=\left(\mu_{*}, \rho_{*}\right)$. Then for $\rho=\rho_{*}$, the value $K^{-1}=\phi(1+\beta)$ is bifurcating if

$$
K_{*}^{-1}=\frac{2 \sqrt{\rho_{*} \mu_{*}}}{\sqrt{\alpha}} .
$$

As $\alpha=1 / 9$ under partial collusion and $\alpha=1 / 8$ under full collusion, this implies

$$
K_{* \text { partial }}^{-1}=6 \sqrt{\rho_{*} \mu_{*}}>4 \sqrt{2} \sqrt{\rho_{*} \mu_{*}}=K_{* \text { full }}^{-1} .
$$

This proves the proposition.

\section{A.5 Proof of Propositions 6 and 5}

We want to compare, for a given parameter combination, the full collusion situation $\alpha=\frac{1}{8}$, and the partial collusion situation $\alpha=\frac{1}{9}$. Performing the scaling to $(c, u)$ variables and $(\mu, \rho)$ parameters, this reduces to comparing the partial collusion situation $\left(\mu_{1}, \rho\right)$ with the full collusion situation $\left(\mu_{2}, \rho\right)$, where the $\mu_{i}$ are related as

$$
\mu_{2}=\frac{9}{8} \mu_{1}
$$

Denote by $u_{f}^{i}, i=1,2$ the corresponding policy functions, and recall that their graphs are locally equal to a portion of a trajectory of (37)-(38), with $u$ replaced by $u_{1}$ or $u_{2}$, depending on whether $\mu=\mu_{1}$ or $\mu=\mu_{2}$. Invoking the chain rule as in (50), we can derive a differential equation for $u_{i}=u_{f}^{i}$ as follows:

$$
\frac{\mathrm{d} u_{i}}{\mathrm{~d} c}=\frac{\dot{u}_{i}}{\dot{c}}=\frac{\rho\left(u_{i}-4 \mu c(1-c) \chi\right)}{c\left(1-u_{i}\right)}
$$

here, we have written $\chi=\chi(c)$ for brevity. This is a first order non-autonomous differential equation, with singularities at $c=0$ and $u_{i}=1$.

Writing $\Delta \mu=\mu_{2}-\mu_{1}$ and $\Delta u=u_{2}-u_{1}$, the difference $\Delta u$ satisfies the following 
differential relation:

$$
\begin{aligned}
\frac{\mathrm{d} \Delta u}{\mathrm{~d} c}= & \frac{\rho\left(u_{2}-4 \mu_{2} c(1-c) \chi\right)}{c\left(1-u_{2}\right)}-\frac{\rho\left(u_{1}-4 \mu_{1} c(1-c) \chi\right)}{c\left(1-u_{1}\right)} \\
= & \frac{\rho\left(1-u_{1}\right)\left(u_{2}-4 \mu_{2} c(1-c) \chi\right)}{c\left(1-u_{1}\right)\left(1-u_{2}\right)}-\frac{\rho\left(1-u_{2}\right)\left(u_{1}-4 \mu_{1} c(1-c) \chi\right)}{c\left(1-u_{1}\right)\left(1-u_{2}\right)} \\
= & \frac{\rho\left(u_{2}-u_{1} u_{2}-4 c(1-c) \chi\left(\mu_{2}-u_{1} \mu_{2}\right)\right)}{c\left(1-u_{1}\right)\left(1-u_{2}\right)} \\
& \quad-\frac{\rho\left(u_{1}-u_{1} u_{2}-4 c(1-c) \chi\left(\mu_{1}-u_{2} \mu_{1}\right)\right)}{c\left(1-u_{1}\right)\left(1-u_{2}\right)} \\
= & \frac{\rho\left(\Delta u-4 c(1-c) \chi\left(\Delta \mu+u_{2} \mu_{2}-u_{1} \mu_{2}-u_{2} \mu_{2}+u_{2} \mu_{1}\right)\right)}{c\left(1-u_{1}\right)\left(1-u_{2}\right)} \\
= & \frac{\rho\left(\Delta u-4 c(1-c) \chi\left(\Delta \mu+\mu_{2} \Delta u-u_{2} \Delta \mu\right)\right)}{c\left(1-u_{1}\right)\left(1-u_{2}\right)} \\
= & \frac{\rho\left(1-4 \mu_{2} c(1-c) \chi\right)}{c\left(1-u_{1}\right)\left(1-u_{2}\right)} \Delta u-\frac{4 \rho(1-c) \chi}{1-u_{1}} \Delta \mu
\end{aligned}
$$

As $u_{1}$ and $u_{2}$ are known, this relation is of the form

$$
\frac{\mathrm{d} \Delta u}{\mathrm{~d} c}=a(c) \Delta u+b(c),
$$

where $a$ and $b$ are known functions. For

$$
\Delta u\left(c_{0}\right)=\Delta_{0}
$$

the variations of constants formula for the solution reads as

$$
\Delta u(c)=\Delta_{0} \mathrm{e}^{\int_{c_{0}}^{c} a(x) \mathrm{d} x}+\int_{c_{0}}^{c} b(x) \mathrm{e}^{\int_{x}^{c} a(y) \mathrm{d} y} \mathrm{~d} x .
$$

\section{A.5.1 Proof of Proposition 5}

Consider first the situation that there is a value $0 \leq \bar{c} \leq 1$ such that for all $c \in(\bar{c}, 1]$ the optimal trajectories for both the partial and the full collusion case leave the production region through $e_{1}$. As we know that trajectories through $e_{1}$ can be optimal only if they have not crossed the line $u=1$ yet, the term $b$ of the variations of constants formula satisfies

$$
b(c)=-\frac{4 \rho(1-c) \chi}{1-u_{1}} \Delta \mu \leq 0
$$

for $\bar{c}<c \leq 1$. Taking $c_{0}=1$ gives $\Delta_{0}=0$, which implies that

$$
\Delta(c)>0
$$

for all $\bar{c}<c \leq 1$. Hence, R\&D effort under full collusion is always larger than R\&D effort under partial collusion if both lead to eventually leaving the market. 
Next, we consider the situation that there is some $\bar{c}>0$, such that for all $c \in(0, \bar{c})$, the optimal trajectories for both the partial and the full collusion case converge to their respective steady states $e_{-}^{1}=\left(c_{-}^{1}, 1\right)$ and $e_{-}^{2}=\left(c_{-}^{2}, 1\right)$. As $\mu_{2}<\mu_{1}$, it follows that $0<c_{-}^{2}<c_{-}^{1} \leq 1 / 2$. The stable manifold tending to $e_{-}^{2}$ can only leave the region bounded by the parabola $u=\mu_{2} c(1-$ $c)$ and the lines $u=1$ and $c=1 / 2$ through the line segment connecting the points $(1 / 2,1)$ with $\left(1 / 2, \mu_{2}\right)$. It follows that necessarily

$$
u_{2}\left(c_{-}^{1}\right)>u_{1}\left(c_{-}^{1}\right), \quad \text { or equivalently, } \quad \Delta\left(c_{-}^{1}\right)>0 .
$$

We have already established that trajectories tending to either $e_{-}^{1}$ or $e_{-}^{2}$ can only be optimal if they do not cross the line $u=1$. Therefore

$$
b(c)=\frac{4 \rho(1-c) \chi}{u_{1}-1} \Delta \mu>0
$$

if $0<c<\bar{c}$, and the variations of constants formula implies

$$
\Delta(c)>0 \text { for all } \quad c_{-}^{1} \leq c<\bar{c} .
$$

Moreover $u_{1}(c)<1$ if $0<c<c_{-}^{1}$, implying that $b(c)<0$ there. Again using the variations of constants formula, we obtain

$$
\Delta(c)>0 \text { for all } 0<c \leq c_{-}^{1}
$$

as well.

Finally, if the optimal trajectory of the full collusion case converges to $e_{-}^{2}$, whereas the optimal trajectory of the partial collusion case exits the production region through $e_{1}$, we have that the former satisfies $u \geq 1$ and the latter $u \leq 1$.

This proves Proposition 5.

\section{A.5.2 Proof of Proposition 6}

To prove Proposition 6, we again use the fact that the value of the integral $\Pi$ over a trajectory starting at a point $(c, u)$ equals

$$
\begin{aligned}
\Pi(c, u) & =\frac{1}{\rho} H_{\text {control }}(c, u)=\frac{1}{\rho}\left(4 \rho \mu(1-c)^{2} \chi-1+(u-1)^{2}\right) \\
& =h(c)+C(u-1)^{2} .
\end{aligned}
$$

If $c=\hat{c}$ is an indifference point, there are values $\hat{u}^{(1)}>\hat{u}^{(2)}$ such that the trajectories starting at $\left(\hat{c}, \hat{u}^{(i)}\right)$, for $i=1,2$, are both optimal and have both the same value. Note that the trajectory through $\left(\hat{c}, \hat{u}^{(1)}\right)$ goes to the left, and that through $\left(\hat{c}, \hat{u}^{(2)}\right)$ goes to the right. As

$$
\Pi\left(\hat{c}, \hat{u}^{(1)}\right)=\Pi\left(\hat{c}, \hat{u}^{(2)}\right)
$$


it follows that

$$
\left|\hat{u}^{(1)}-1\right|=\left|\hat{u}^{(2)}-1\right| .
$$

Consider a fixed value of $\rho$ and two values $\mu_{1}, \mu_{2}$ of $\mu$ such that $\mu_{2}=(9 / 8) \mu_{1}$; that is, $\left(\mu_{1}, \rho\right)$ describes a partial collusion situation, and $\left(\mu_{2}, \rho\right)$ is the corresponding full collusion situation.

Assume first that there is an indifference point in the partial collusion problem; denote these points as $\hat{c}_{1}$, and the corresponding values of $u$ as

$$
\hat{u}_{1}^{(1)}<\hat{u}_{1}^{(2)} .
$$

We have seen in the proof of Proposition 5 that necessarily the full collusion trajectory going towards $e_{-}^{2}$ is above the partial collusion trajectory going towards $e_{-}^{1}$. Denote its intersection with the line $c=\hat{c}_{1}$ by $\left(\hat{c}_{1}, \hat{u}_{2}^{(1)}\right)$. We have that

$$
\left|\hat{u}_{2}^{(1)}-1\right|>\left|\hat{u}_{1}^{(1)}-1\right|
$$

We argue by contradiction. Assume that the threshold $\hat{c}_{2}$ in the full collusion case exists and is below the threshold in the partial collusion case, then the full collusion trajectory going right, that is, to $e_{1}$, has to intersect the line $c=\hat{c}_{1}$ in a point $\left(\hat{c}_{1}, \hat{u}_{2}^{(2)}\right)$. Moreover, this trajectory has to be optimal at $\hat{c}_{1}$. Using (55), this implies that

$$
\left|\hat{u}_{2}^{(1)}-1\right|<\left|\hat{u}_{2}^{(2)}-1\right| \text {. }
$$

Finally, the full collusion trajectory has to be above the partial collusion trajectory going to $e_{1}$, implying

$$
\left|\hat{u}_{2}^{(2)}-1\right|<\left|\hat{u}_{1}^{(2)}-1\right| .
$$

Combining these inequalities with the fact that $\hat{c}_{1}$ is an indifference point in the partial collusion situation, we arrive at

$$
\left|\hat{u}_{2}^{(1)}-1\right|>\left|\hat{u}_{1}^{(1)}-1\right|=\left|\hat{u}_{1}^{(2)}-1\right|>\left|\hat{u}_{2}^{(2)}-1\right|>\left|\hat{u}_{2}^{(1)}-1\right| .
$$

But this is a contradiction. The proof in situation that the threshold is a repeller is similar and will be omitted. 


\section{References}

[1] AGHION, P. and HOWITT, P. (1992), "A Model of Growth Through Creative Destruction", Econometrica, (60), 323-351.

[2] ARROW, K. J. (1962), "Economic Welfare and the Allocation of Resources for Invention", in Nelson, R.R. (ed.), The Rate and Direction of Inventive Activity: Economic and Social Factors, National Bureau of Economic Research, Conference Series, Princeton: Princeton University Press, 609-625.

[3] BENKARD, C. L. (2004), "A Dynamic Analysis of the Market for Wide-Bodied Commercial Aircraft", Review of Economic Studies, 71, 581-611.

[4] BESANKO, D., DORASZELSKI, U., KRYUKOV, Y. and SATTERTHWAITE, M. (2010), "Learning-by-Doing, Organizational Forgetting, and Industry Dynamics", Econometrica, 78, 453-508.

[5] BLOOM, N., SCHANKERMAN, M. and VAN REENEN, J. (2013), "Identifying Technology Spillovers and Product Market Rivalry", Econometrica, 81, 1347-1393.

[6] BOND, S., HARHOFF, D. and VAN REENEN, J. (2005), "Investment, R\&D and Financial Constraints in Britain and Germany", Annales d'Economie et de Statistique, 433-460.

[7] BRODLEY, J. (1990), "Antitrust Law and Innovation Cooperation", Journal of Economic Perspectives, 4, 97-112.

[8] CABRAL, L. (2000), "R\&D Cooperation and Product Market Competition", International Journal of Industrial Organization, 18, 1033-1047.

[9] CALOGHIROU, Y., IOANNIDES, S. and VONORTAS, N. S. (2003), "Research Joint Ventures", Journal of Economic Surveys, 17, 541-570.

[10] CELLINI, R. and LAMBERTINI, L. (2005), "R\&D Incentives and Market Structure: Dynamic Analysis", Journal of Optimization Theory and Applications, 126, 85-96.

[11] CELLINI, R. and LAMBERTINI, L. (2009), "Dynamic R\&D with Spillovers: Competition vs Cooperation”, Journal of Economic Dynamics \& Control, 33, 568-582.

[12] COHEN, W. M. and LEVINTHAL, D. A. (1989), "Innovation and Learning: The Two Faces of R\&D”, The Economic Journal, 99, 569-596.

[13] COOPER, A. C. and SCHENDEL, D. (1976), "Strategic Responses to Technological Threats", Business Horizons, 19, 61-69.

[14] D'ASPREMONT, C. and JACQUEMIN, A. (1988), "Cooperative and Noncooperative R\&D in Duopoly with Spillovers", The American Economic Review, 78, 1133-1137. 
[15] DUSO, T., RÖLLER, L. H. and SELDESLACHTS, J. (2014), "Collusion Through Joint R\&D: An Empirical Assessment”, Review of Economics and Statistics, 96, 349-370.

[16] FISHER, F. M. (1990), Discussion of Michael L. Katz and Janusz A. Ordover, "R\&D Cooperation and Competition", Brookings Papers on Economic Activity, Microeconomics 1990, 192-194.

[17] FLEMING, W. H. and SONER, H. M. (2006), Controlled Markov Processes and Viscosity Solutions (New York: Springer).

[18] GEROSKI, P. A. (1992), "Vertical Relations Between Firms and Industrial Policy", Economic Journal, 102, 138-147

[19] GOEREE, M. S. and HELLAND, E. (2008), "Do Research Joint Ventures Serve a Collusive Function?" Institute for Empirical Research in Economics, University of Zurich, Working Paper No. 448.

[20] GRASS, D., CAULKING, J. P., FEICHTINGER, G., TRAGLER, G. and BEHRENS, D. A. (2008), Optimal Control of Nonlinear Processes (Springer-Verlag).

[21] GREEN, J. R. and SCOTCHMER, S. (1995), "On the Division of Profit in Sequential Innovation”, The RAND Journal of Economics, 26, 20-33.

[22] GREENLEE, P. and CASSIMAN, B. (1999), "Product Market Objectives and the Formation of Research Joint Ventures", Managerial and Decision Economics, 20, 115 -130.

[23] GREENSPAN, A. (1962), Antitrust (New York: Nathaniel Branden Institute).

[24] GROSSMAN, G. M. and HELPMAN, E. (1991), "Quality Ladders in the Theory of Growth”, The Review of Economic Studies, 58, 43-61.

[25] GROSSMAN, G. M. and SHAPIRO, C. (1986), "Research Joint Ventures: An Antitrust Analysis", Journal of Law, Economics \& Organization, 2, 315-337.

[26] GUCKENHEIMER, J. and HOLMES, P. (1986), Nonlinear Oscillations, Dynamical Systems and Bifurcations of Vector Fields (New York: Springer).

[27] HALL, B. H. and LERNER, J. (2010), “The Financing of R\&D and Innovation”, In: HALL, B. H. and ROSENBERG, N. (Eds.), Handbook of the Economics of Innovation (North-Holland).

[28] HALL, B. H., MAIRESSE, J., BRANSTETTER, L. and CREPON, B. (1999), "Does Cash Flow Cause Investment and R\&D? An Exploration Using Panel Data for French, Japanese and United States Firms in the Scientific Sector", In: AUDRETSCH, D., THURIK, A. R. (Eds.), Innovation, Industry Evolution and Employment (Cambridge, UK: Cambridge University Press). 
[29] HARHOFF, D. (1998), "Are There Financing Constraints for R\&D and Investment in German Manufacturing Firms?”, Annales d'Economie et de Statistique, 421-456.

[30] HERNAN, R., MARIN, P. L. and SIOTIS, G. (2003), "An Empirical Evaluation of the Determinants of Research Joint Venture Formation", Journal of Industrial Economics, 51, $75-89$.

[31] HIMMELBERG, C. P. and PETERSEN, B. C. (1994), "R\&D and Internal Finance: A Panel Study of Small Firms in High-Tech Industries", Review of Economics and Statistics, 76, $38-51$.

[32] HINLOOPEN, J. (2000), "Strategic R\&D Co-operatives", Research in Economics, 54, $153-185$.

[33] HINLOOPEN, J. (2003), "R\&D Efficiency Gains Due to Cooperation", Journal of Economics, 80, 107-125.

[34] HINLOOPEN, J., SMRKOLJ, G. and WAGENER, F. O. O. (2013), "From Mind to Market: A Global, Dynamic Analysis of R\&D”, Journal of Economic Dynamics \& Control, 37, 2729-2754.

[35] HOWELLS, J. (2002), "The Response of Old Technology Incumbents to Technological Competition - Does the Sailing Ship Effect Exist?”, Journal of Management Studies, 39, 887-906.

[36] JACQUEMIN, A. (1988), "Cooperative Agreements in R\&D and European Antitrust Policy”, European Economic Review, 32, 551-560.

[37] KAMIEN, M. I., MULLER, E. and ZANG, I. (1992), "Research Joint Ventures and R\&D Cartels", The American Economic Review, 82, 1293-1306.

[38] KISELEVA, T. and WAGENER, F. O. O. (2010), "Bifurcations of Optimal Vector Fields in the Shallow Lake Model”, Journal of Economic Dynamics \& Control, 34, 825-843.

[39] KISELEVA, T. and WAGENER, F. O. O. (2015), "Bifurcations of Optimal Vector Fields", Mathematics of Operations Research, 40, 24-55.

[40] KOVAC, E., VINOGRADOV, V. and ZIGIC, K. (2010), “Technological Leadership and Persistence of Monopoly Under Endogenous Entry: Static Versus Dynamic Analysis", Journal of Economic Dynamics \& Control, 34, 1421-1441.

[41] LAMBERTINI, L. and MANTOVANI, A. (2009), "Process and Product Innovation by a Multiproduct Monopolist: A Dynamic Approach", International Journal of Industrial Organization, 27, 508-518. 
[42] LAMBERTINI, L. and MANTOVANI, A. (2010), "Process and Product Innovation: A Differential Game Approach to Product Life Cycle", International Journal of Economic Theory, 6, 227-252.

[43] LAMBERTINI, L., PODDAR, S. and SASAKI, D. (2002), "Research Joint Ventures, Product Differentiation and Price Collusion", International Journal of Industrial Organization, 20, 829-854.

[44] LEE, T. and WILDE, L. L. (1980), "Market Structure and Innovation: A Reformulation”, The Quarterly Journal of Economics, 94, 429-436.

[45] LOURY, G. C. (1979), "Market Structure and Innovation", The Quarterly Journal of Economics, 93, 395-410.

[46] MARTIN, S. (1995), "R\&D Joint Ventures and Tacit Product Market Collusion”, European Journal of Political Economy, 11, 733-741.

[47] MARTIN, S. (1997), "Public Policies Toward Cooperation in Research and Development: The European Union, Japan, the United States”, in Waverman, L., Comanor, W. and Goto, A. (1997), Competition Policy in the Global Economy (London: Routledge), 245-288.

[48] MICHEL, P. (1982), "On the Transversality Condition in Infinite Horizon Optimal Problems", Econometrica, 50, 975-985.

[49] MIYAGIWA, K. (2009), "Collusion and Research Joint Ventures", Journal of Industrial Economics, 57, 768-784.

[50] PFEFFER, J. and NOWAK, P. (1976), "Patterns of Joint Venture Activity: Implications for Antitrust Policy”, Antitrust Bulletin, 21, 315-339.

[51] PIGA, C. A. and ATZENI, G. (2007), "R\&D Investment, Credit Rationing and Sample Selection”, Bulletin of Economic Research, 59, 149-178.

[52] QUIRMBACH, H. C. (1993), "R\&D: Competition, Risk, and Performance", The RAND Journal of Economics, 24, 157-197.

[53] REINGANUM, J. F. (1989), "The Timing of Innovation: Research, Development, and Diffusion”, Handbook of Industrial Organization, 1, 849-908.

[54] RÖLLER, L. H., TOMBAK, M. and SIEBERT, R. (2007), "Why Firms Form (or do not Form) RJVS”, The Economic Journal, 117, 1122-1144.

[55] SALANT, S. W. and SHAFFER, G. (1998), "Optimal Asymmetric Strategies in Research Joint Ventures”, International Journal of Industrial Organization, 16, 195-208.

[56] SCHERER, F. M. (1980), Industrial Market Structure and Economic Performance (Chicago: Rand McNally College Publishing Co.). 
[57] SCHUMPETER, J. (1934), The Theory of Economic Development: An Inquiry into Profits, Capital, Credit, Interest, and the Business Cycle, translated by Opie, R. (Cambridge: Harvard University Press).

[58] SCHWARTZMAN, D. (1976), Innovation in the Pharmaceutical Industry (Baltimore: John Hopkins University Press).

[59] SOTOMAYOR, J. (1973), "Generic Bifurcations of Dynamical Systems”, In Peixoto, M. M. (ed.), Dynamical Systems (New York: Academic Press), 549-560.

[60] SUETENS, S. (2008), "Does R\&D Cooperation Facilitate Price Collusion? An Experiment", Journal of Economic Behaviour \& Organization, 66, 822-836.

[61] TIROLE, J. (1988), The Theory of Industrial Organization (MIT Press).

[62] YEUNG, D.W.K. and PETROSYAN, L.A. (2004), "Subgame Consistent Cooperative Solutions in Stochastic Differential Games", Journal of Optimization Theory and Applications, 120, 651-666.

[63] WAGENER, F. O. O. (2003), "Skiba Points and Heteroclinic Bifurcations, with Applications to the Shallow Lake System”, Journal of Economic Dynamics \& Control, 27, 1533-1561. 